\title{
Sex-specific brain erythropoietin regulation of mouse metabolism and hypothalamic inflammation
}

\author{
Soumyadeep Dey, ${ }^{1}$ Zhenzhong Cui, ${ }^{2}$ Oksana Gavrilova, ${ }^{2}$ Xiaojie Zhang, ${ }^{3}$ Max Gassmann, ${ }^{4,5}$ \\ and Constance T. Noguchi' \\ ${ }^{1}$ Molecular Medicine Branch, ${ }^{2}$ Mouse Metabolism Core Laboratory, and ${ }^{3}$ Laboratory of Animal Sciences Section, National \\ Institute of Diabetes and Digestive and Kidney Diseases, NIH, Bethesda, Maryland, USA. ${ }^{4}$ Institute of Veterinary \\ Physiology and Zurich Center for Integrative Human Physiology, University of Zurich, Zurich, Switzerland. ${ }^{5}$ Universidad \\ Peruana Cayetano Heredia, Lima, Peru.
}

The blood hormone erythropoietin (EPO), upon binding to its receptor (EpoR), modulates highfat diet-induced (HFD-induced) obesity in mice, improves glucose tolerance, and prevents white adipose tissue inflammation. Transgenic mice with constitutive overexpression of human EPO solely in the brain ( $\operatorname{Tg} 21)$ were used to assess the neuroendocrine EPO effect without increasing the hematocrit. Male Tg21 mice resisted HFD-induced weight gain; showed lower serum adrenocorticotropic hormone, corticosterone, and C-reactive protein levels; and prevented myeloid cell recruitment to the hypothalamus compared with WT male mice. HFD-induced hypothalamic inflammation (HI) and microglial activation were higher in male mice, and Tg21 male mice exhibited a lower increase in HI than WT male mice. Physiological EPO function in the brain also showed sexual dimorphism in regulating HFD response. Female estrogen production blocked reduced weight gain and HI. Targeted deletion of EpoR gene expression in neuronal cells worsened HFDinduced glucose intolerance in both male and female mice but increased weight gain and $\mathrm{HI}$ in the hypothalamus in male mice only. Both male and female $\mathrm{Tg} 21$ mice kept on normal chow and HFD showed significantly improved glycemic control. Our data indicate that cerebral EPO regulates weight gain and $\mathrm{HI}$ in a sex-dependent response, distinct from EPO regulation of glycemic control, and independent of erythropoietic EPO response.

Conflict of interest: The authors have declared that no conflict of interest exists.

Copyright: () 2020, American Society for Clinical Investigation.

Submitted: October 7, 2019

Accepted: February 12, 2020

Published: March 12, 2020.

Reference information: JCI Insight. 2020;5(5):e134061.

https://doi.org/10.1172/jici.

insight.134061.

\section{Introduction}

Erythropoietin (EPO), the blood hormone produced primarily in fetal liver and adult kidneys upon hypoxic stimulation, is required for erythrocyte propagation and maturation and functions through binding to its specific transmembrane receptor (EpoR) (1). Recombinant human EPO is used clinically to treat anemia associated with chronic kidney disease, cancer chemotherapy, or HIV antiretroviral therapy. In mice, targeted deletion of Epo or EpoR gene results in embryonic lethality due to severe anemia $(2,3)$. EpoR signaling in hematopoietic cells is determined by EPO concentration and EpoR cell surface expression level. Beyond erythroid progenitor/precursor cells, EpoR expression and EPO activity has been detected in nonhematopoietic cells $(4,5)$, including macrophages $(6,7)$, osteoclasts (8), microglial cells (9), neurons (10), endothelial cells (11), and adipocytes (12). This produces several nonerythroid physiological effects of EPO.

Erythroid transgenic rescue of $E p o R$-knockout mice (termed $\triangle E p o R_{E}$ mice) restores early development and erythropoiesis but results in obesity by 4 weeks of age (12). $\triangle E p o R_{E}$ mice develop metabolic syndrome with increased fat, glucose intolerance, and insulin resistance, resulting from low energy expenditure due primarily to effects in white adipose tissue (WAT) and the hypothalamus. $\triangle E p o R_{E}$ mice show increased white fat mass and adipocyte number (12), reduced hypothalamic expression of anorexigenic neuropeptide pro-opiomelanocortin (POMC) (13), and higher serum adrenocorticotropic hormone (ACTH) due to deregulated secretion from the pituitary gland (14). Conversely, exogenous EPO treatment in WT mice can prevent diet-induced obesity (DIO), improve glucose tolerance, reduce food intake $(12,15,16)$, and shift WAT-associated macrophages from a proinflammatory to an antiinflammatory phenotype (6). Male mice are more susceptible to DIO than 
female mice, and EPO is more effective in preventing weight and fat gain in males, indicating sexually dimorphic EPO regulation of metabolism that may also occur in humans $(17,18)$.

EPO is also synthesized in the brain, mainly by astrocytes and neurons, is hypoxia inducible, and is associated with neural progenitor cell proliferation and differentiation (19-21). EPO has been associated with neurogenesis, neuroprotection, and neural progenitor cell migration (21-24). In the hypothalamus, the arcuate nucleus is a major central neuroendocrine regulation center controlling energy homeostasis and EpoR expression colocalizes with POMC-producing neurons $(12,13)$. EPO can directly induce POMC expression through STAT3 signaling, and lack of EpoR reduces POMC, contributing to EPO-mediated metabolic effects. The hypothalamus communicates with the pituitary gland and processes signals from peripheral energy stores to maintain energy homeostasis through secretion of endocrine hormones (25). Chronic overnutrition resulting in DIO puts excessive stress on this regulation, and high circulating levels of free fatty acids, glucose, and amino acids result in a hypothalamus inflammatory response, analogous to peripheral inflammation in WAT and liver (26). Microglial cells, specialized macrophage cells in the brain, become activated and release cytokines that initially are protective but chronically cause hypothalamic dysfunction, leading to obesity and glucose intolerance (27).

Here, we investigate the effect of cerebral EPO on the brain/hypothalamus during DIO that in turn mediates the EPO metabolic regulation. To this end, we used transgenic Tg21 mice that present with constitutive brain-specific overexpression of human EPO in an oxygen-independent manner and without an increase in EPO-stimulated erythropoiesis (28), EPO infusion in brain in WT mice, and knockout of cerebral $E p o R$ gene expression to assess the protective potential of cerebral EPO during high-fat diet (HFD) feeding.

\section{Results}

Transgenic expression of cerebral EPO prevents weight gain, increases energy expenditure, and improves glucose tolerance and insulin sensitivity in male mice. DIO was reduced in male Tg21 mice with elevated brain EPO levels. During 8 weeks of HFD, beginning at 5 weeks of age, cumulated body weight and fat mass in male mice were markedly lower in $\mathrm{Tg} 21$ mice compared with those in WT control male mice (Figure 1, A and B). Weight gain and fat mass increased by $90 \%$ and $600 \%$, respectively, in male WT mice compared with $60 \%$ and $180 \%$ in $T g 21$ mice, respectively, with a comparable increase in lean mass (Figure 1C). In contrast, female $\operatorname{Tg} 21$ and WT mice did not have different body weight gain and fat accumulation after HFD feeding (Figure 1, D-F). Male Tg21 mice showed a trend toward eating more and a significantly higher energy expenditure (Figure $1, \mathrm{G}$ and $\mathrm{H}$ ).

Glucose tolerance measured during normal chow diet (NCD) and after 3 weeks of HFD feeding was improved in both male and female Tg21 mice compared with WT mice (Figure 1, I, J, L, and M). Moreover, while WT and Tg21 male and female mice on NCD did not show any difference in fasting blood glucose levels, glucose levels were higher in WT mice during HFD feeding ( 0 -minute time point, Figure 1 , I, J, L, and M). Insulin levels were elevated in WT mice fed HFD, suggesting that insulin sensitivity was reduced after 3 weeks of HFD, but not in Tg21 mice (Figure 1, K and N). These data suggest that, when fed with HFD, WT male mice were more susceptible to DIO and showed lower energy expenditure than $T g 21$ male mice, while both male and female $\mathrm{Tg} 21$ mice showed improved glucose tolerance during NCD and HFD and better insulin sensitivity on HFD than WT mice.

Tg21 male mice are protected from DIO-induced hypothalamus inflammation and microglial cell activation. DIO is associated with chronic low-grade hypothalamus inflammation that occurs within the first few days after HFD feeding, induces hypothalamic activation of microglial cells, and increases proinflammatory cytokines (27). Accordingly, we quantified inflammatory cytokine marker TNF- $\alpha$, microglial cell marker Iba1, and activated microglial cell marker CD68 to assess the EPO-regulated response in the mediobasal hypothalamus (MBH) of male mice. On NCD, the number of TNF- $\alpha^{+}$cells was very small, but this number was more pronounced in WT mice compared with $T g 21$ male mice (Figure 2, A and C). On HFD feeding, the numbers increased in both WT and Tg21 male mice and became 3-fold higher in WT animals compared with Tg21 animals (Figure 2, B and C). Similarly, the number of microglial cells was higher in WT mice on NCD (Figure 2, A and D). With DIO, Iba $1^{+}$cells increased in both WT and Tg21 mice and became 2-fold higher in WT mice compared with $T g 21$ mice (Figure 2, B and D). Quantification of Iba1 ${ }^{+}$staining also showed higher staining density in WT mice compared with $T g 21$ mice (Figure 2, E and F). CD68 was also more abundant in the MBH region of HFD-fed WT mice compared with HFD-fed Tg21 mice (Figure 2, G and $\mathrm{H}$ ). Moreover, during DIO, expression of 3 additionally analyzed inflammatory genes ( $C c l 2$, Il6, and $I l 1 \beta$ ) increased markedly in the hypothalamus of WT male mice, with little or no change in the $T g 21$ hypo- 
$\mathbf{A}_{50}$

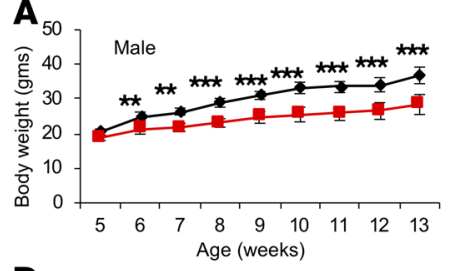

D

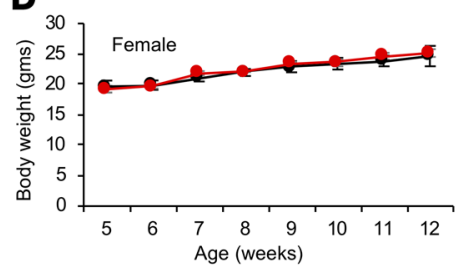

G

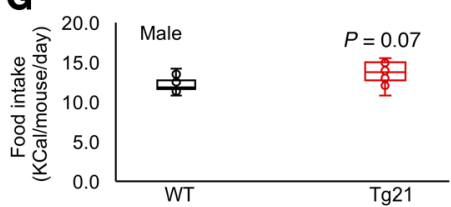

I

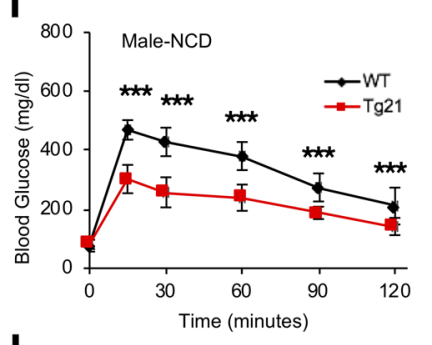

$\mathbf{L}$

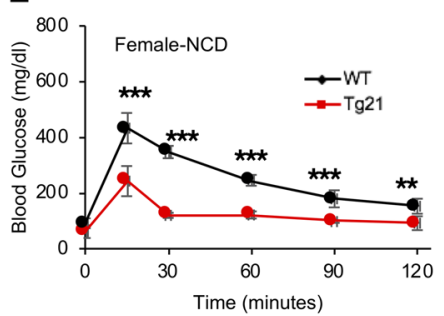

B

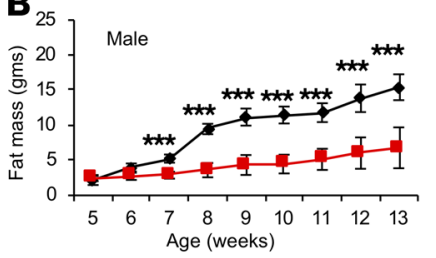

E

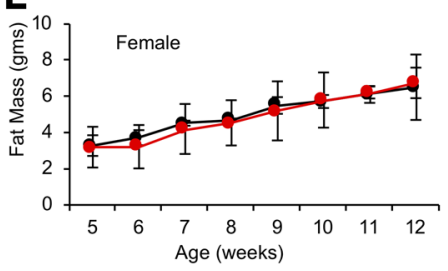

H
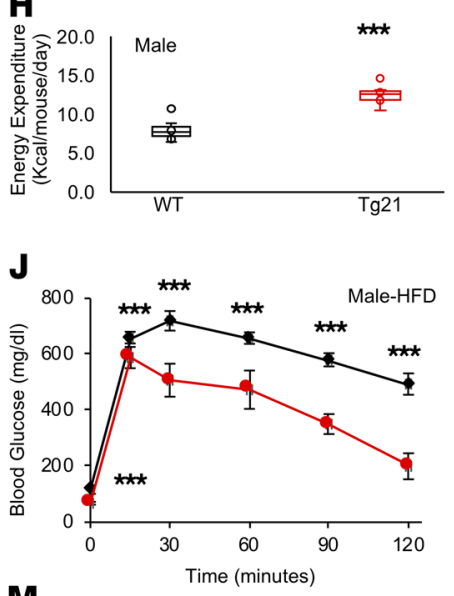

M

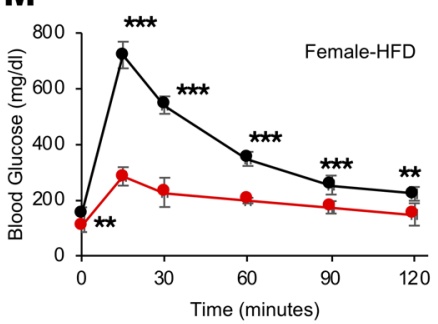

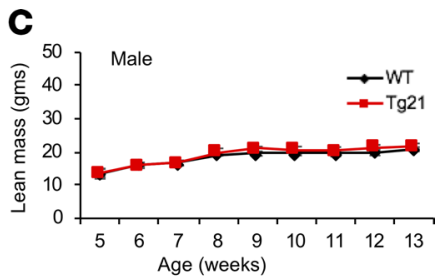

$\mathbf{F}$

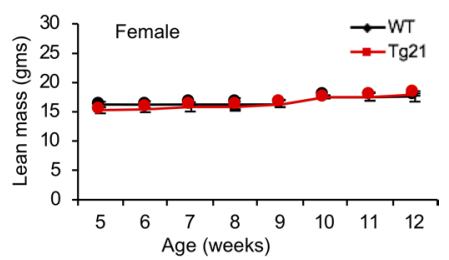

$\mathbf{K}$
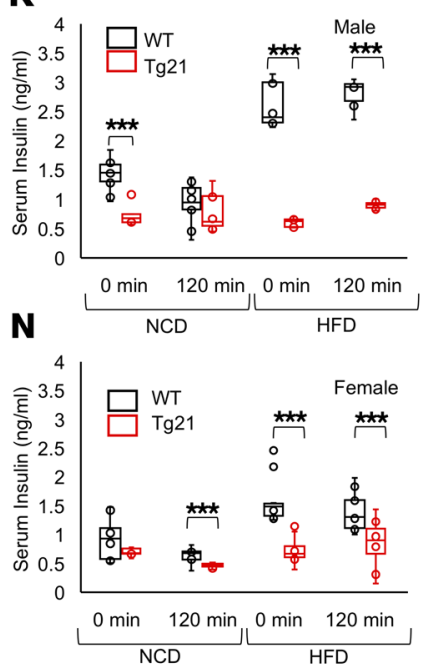

Figure 1. During DIO, Tg21 mice show reduced weight gain/fat mass accumulation and increased energy expenditure in male mice and improved glucose utilization in male and female mice. (A-F) Body weight ( $\mathbf{A}$ and $\mathbf{D}$ ), fat mass (B and $\mathbf{E}$ ), and lean mass (C and $\mathbf{F})$ in HFD-fed Tg21 and WT mice beginning at 5 weeks of age in male mice (A-C) and female mice (D-F). ( $\mathbf{G}$ and $\mathbf{H})$ Daily average food intake (G) and daily energy expenditure (H) at 5-8 weeks of age on HFD feeding in male Tg21 and WT mice. (I-N) Glucose tolerance test (GTT) and serum insulin in males (I-K) and females (L-N) in Tg21 (red line) and WT (black line) mice during NCD (age, 5 weeks, $\mathbf{I}$ and $\mathbf{L}$ ) and after 3 weeks of HFD (age, 8 weeks, J and $\mathbf{M}$ ). Serum insulin measurements at the beginning ( 0 minutes) and end (120 minutes) of GTT during NCD and HFD in male (K) and female (N) mice. Each graph is representative of at least 3 independent experiments; $n=8-10$ for each data point. For line graph, each data point represents mean $\pm S D$. In box-and-whisker plots, bounds denote the 25 th to 75 th percentile, the lines represent the medians, and whiskers indicate the range from minimum to maximum values and includes outliers. ${ }^{* *} P<0.01,{ }^{* *} P<0.001$ ( 2 -way ANOVA).

thalamus (Figure 2I). Importantly, these changes in hypothalamus inflammation are sexually dimorphic. In female WT and $T g 21$ mice, TNF- $\alpha^{+}$and $\mathrm{Iba}^{+}$cell numbers were very low during $\mathrm{NCD}$, with comparable increases during HFD feeding, and there was no significant difference in $C c l 2$, Il6, and Il1 $\beta$ relative gene expression in the hypothalamus (Supplemental Figure 1; supplemental material available online with this article; https://doi.org/10.1172/jci.insight.134061DS1).

As DIO induces rapid infiltration of blood-derived peripheral myeloid cells into the hypothalamus (29), we assessed recruitment of peripheral myeloid cells into the MBH. P2Y12 is a very specific marker for microglial cells, and its expression is reduced in activated microglial cells (30). During NCD, the number of P2Y12 cells was moderately higher in male WT mice (Figure 3, A and C), while during DIO, these cells decreased in WT mice but increased in $\operatorname{Tg} 21$ mice to almost double the WT number (Figure 3, A and C). CD169 is a mark- 

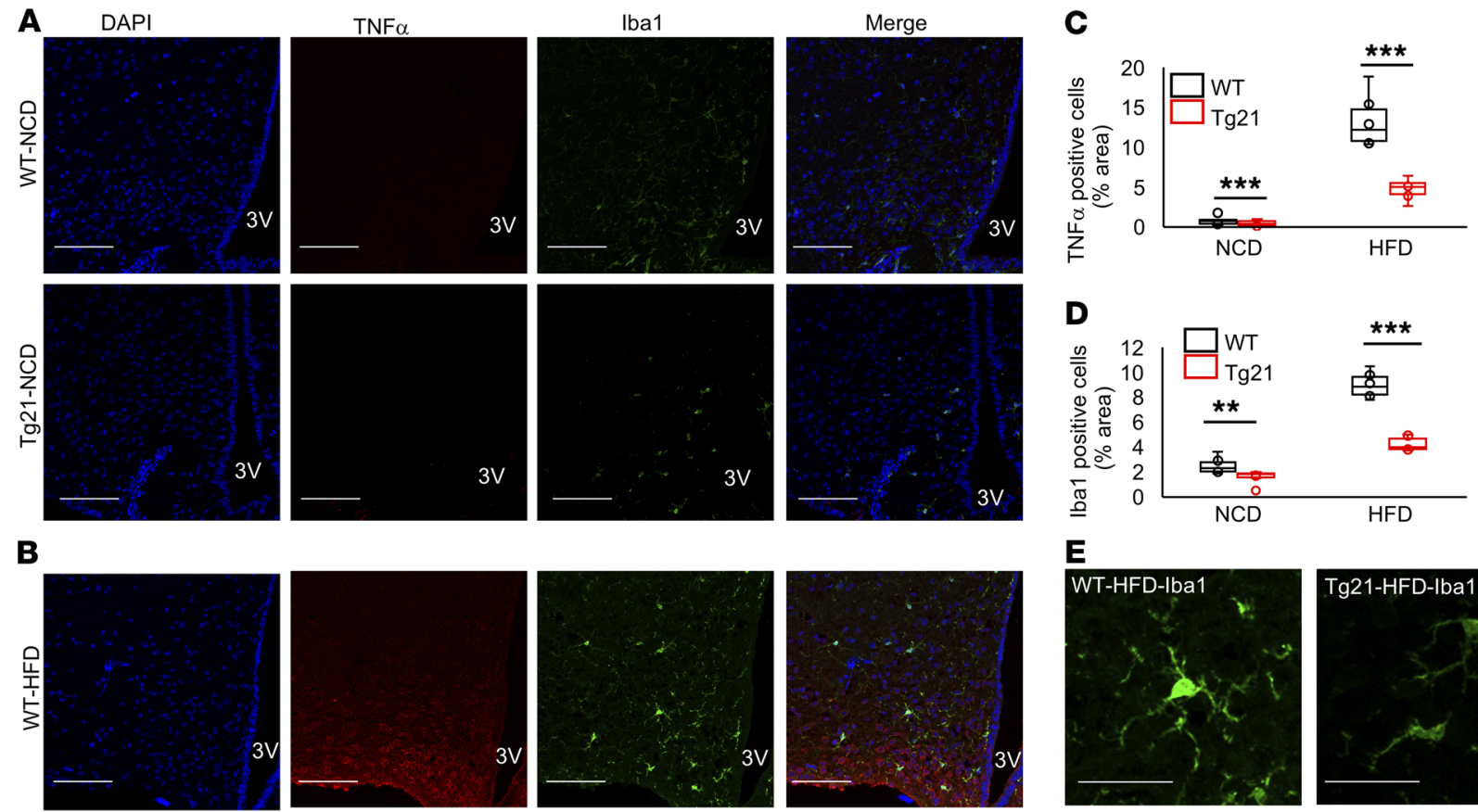

\section{$\mathbf{E}$}
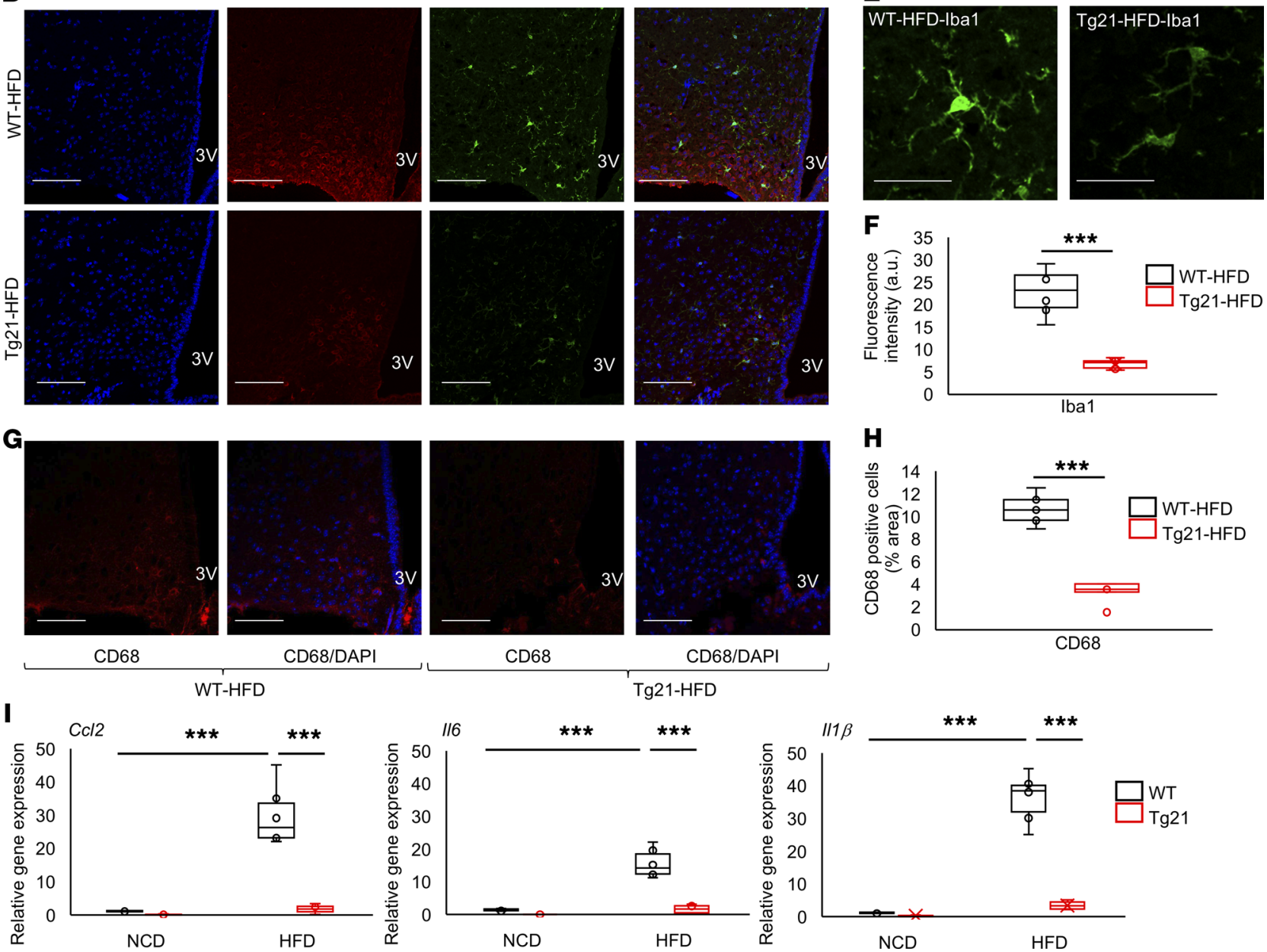

Figure 2. Reduced hypothalamus inflammatory response and microglial cell activation in Tg21 male mice compared with WT male mice. (A and B) Representative hypothalamic sections of age-matched (8 weeks) male WT and Tg21 mice during NCD (A) or after 3 weeks of HFD feeding (B) stained for the nuclear marker, DAPI; inflammatory marker, TNF- $\alpha$; and microglial cell marker, Iba1. 3V, third ventricle. Scale bar: $100 \mu \mathrm{m}$. Original magnification, $\times \mathbf{4 0}$. (C and $\mathbf{D})$ Quantification of $\mathbf{A}$ and $\mathbf{B}$ to denote TNF- $\alpha^{+}$and Iba1 ${ }^{+}$cells. (E and $\left.\mathbf{F}\right)$ Representative fields of view in HFD-fed WT and Tg21 mice (scale bar: $20 \mu \mathrm{m}$ ) (E), analyzed for quantification of fluorescence intensity of Iba1 (F). (G and $\mathbf{H})$ Representative hypothalamic sections of age-matched (8 weeks) male WT and Tg21 mice after 3 weeks of HFD feeding stained for activated microglial cell markers, CD68 and DAPI (scale bar: $100 \mu \mathrm{m}$; original magnification, $\times 40)(\mathbf{G})$, and quantification of CD68+ cells $(\mathbf{H})$. Each image is representative of $n=8-10 /$ group. (I) Gene expression of markers $C \mathrm{Cl} 2, \| 6$, and $I / 1 \beta$ in the hypothalamus of age-matched (8 weeks) male Tg21 and WT mice on NCD or after 3 weeks of HFD, as determined by quantitative RT-PCR, normalized to WT control on NCD and adjusted to Gapdh gene expression ( $n=8-10 /$ group). In box-and-whisker plots, bounds denote the 25 th to 75 th percentile, the lines represent the medians, and whiskers indicate the range from minimum to maximum values and includes outliers. ${ }^{* *} P<0.01,{ }^{* * *} P<0.001$ ( 2 -way ANOVA). 
A
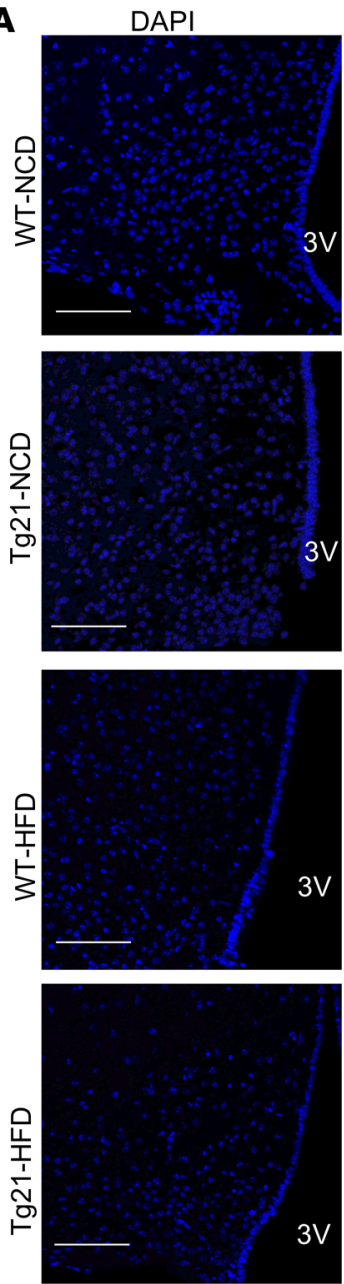

$3 \mathrm{~V}$
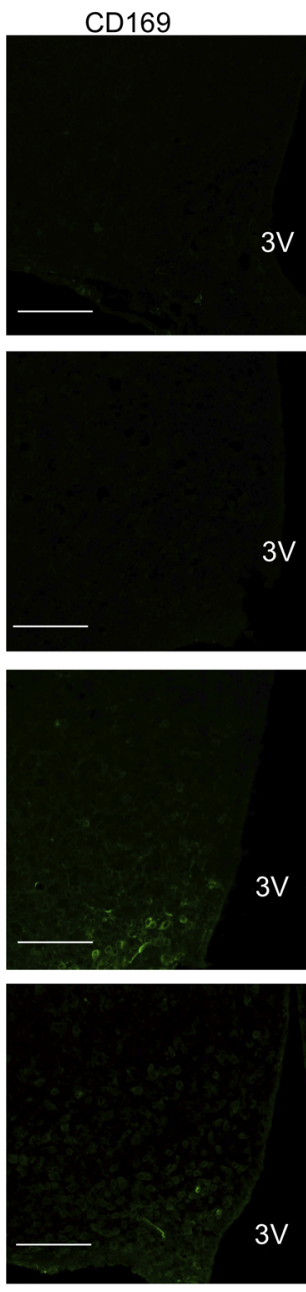

P2Y12
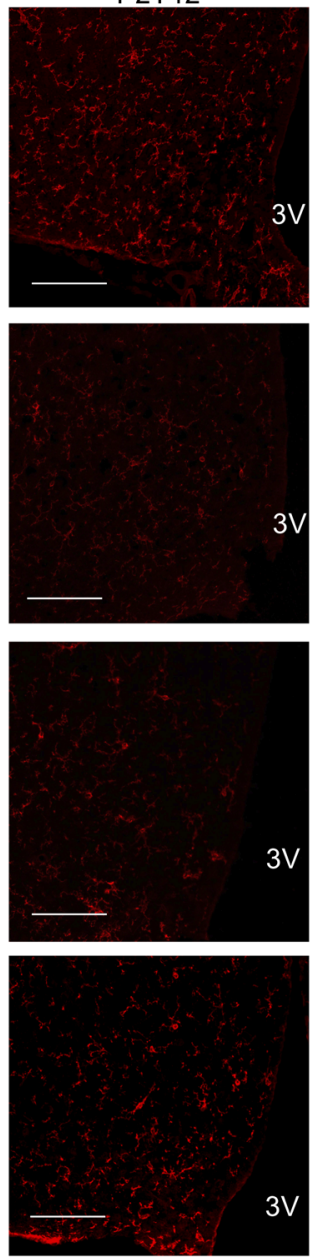

$3 V$

$\mathrm{V}$
Merge
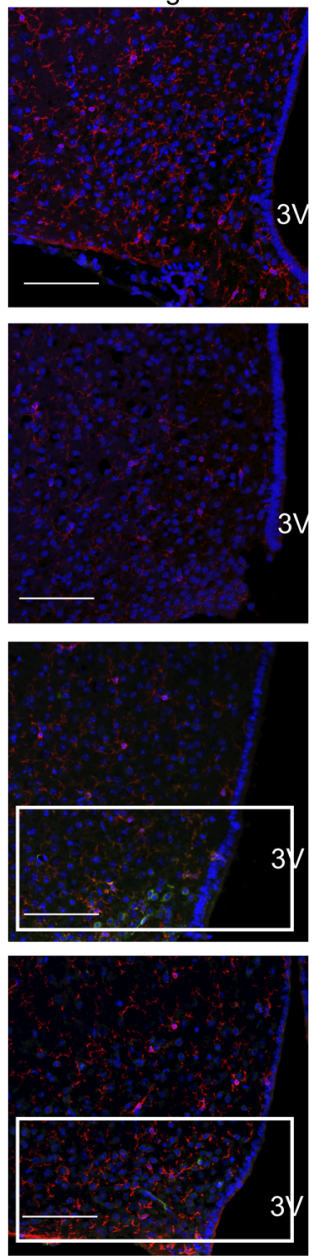

B

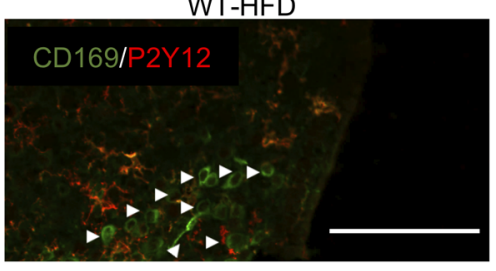

C

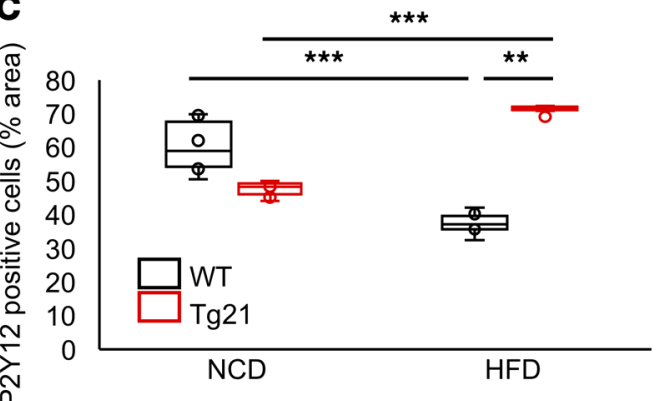

Tg21-HFD

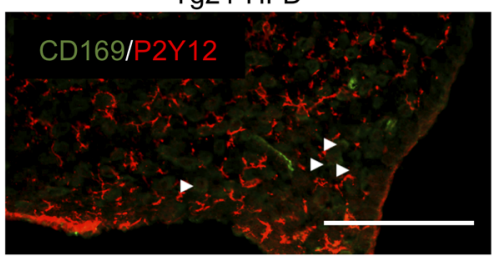

D

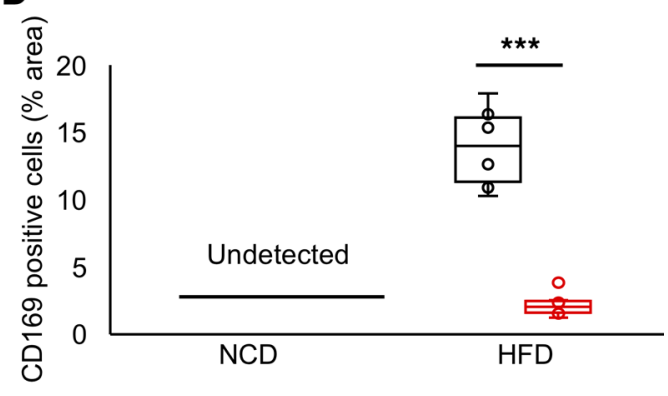

Figure 3. Nonactivated P2Y12+ microglial cells in male Tg21 mice are reduced under NCD and increased on HFD feeding but decreased in male WT mice on HFD feeding, with an increase in peripheral CD169+ cell recruitment. (A) Representative hypothalamic sections of age-matched ( 8 weeks) male Tg21 and WT mice during NCD or after 3 weeks of HFD feeding stained for the nuclear marker, DAPI; peripheral macrophage marker, CD169; and nonactivated microglial marker, P2Y12, in MBH. (B) Visualization of boxed areas from A without DAPI staining and with arrowheads pointing to CD169+ cells. (C and D) Quantification of A. Scale bar: $100 \mu \mathrm{m}$. Original magnification, $\times 40$. 3V, third ventricle. Each image is representative of $n=8-10 / g r o u p$. In box-and-whisker plots, bounds denote the 25 th to 75 th percentile, the lines represent the medians, and whiskers indicate the range from minimum to maximum values and includes outliers. ${ }^{* *} P<0.01,{ }^{* *} P<0.001$ (2-way ANOVA). 
er for myeloid monocyte-derived cells recruited from the periphery and not expressed by resident microglial cells (31). Few or no CD169 ${ }^{+}$cells were detected during NCD; with the development of DIO, the number of CD169+ cells increased in male mice, reaching 5-fold higher or more in WT mice compared with $\operatorname{Tg} 21$ mice (Figure 3, A, B, and D). Again, these changes are sex specific; in females, the number of P2Y12 ${ }^{+}$cells was comparable and $\mathrm{CD} 169^{+}$staining was almost undetectable in WT and Tg21 mice (Supplemental Figure 2).

HFD-induced weight gain, glucose intolerance, and hypothalamic inflammation are regulated by endogenous physiological EPO/EpoR signaling. Cerebral EPO expression in transgenic $T g 21$ mice is approximately 4-fold compared with WT controls (28). To study if physiological EPO levels and signaling via EpoR in the brain, especially in neuronal cells, can regulate HFD-induced metabolic changes in mice, we knocked out EpoR gene expression in neuronal cell precursors. This was achieved by mating chimeric EpoR-floxed mice with mice containing a Cre-recombinase gene controlled by the nestin promoter (32). Glial EpoR expression was expected to be unchanged. Ablation of EpoR gene expression in nestin-Cre;EpoR ${ }^{\text {loxP/loxP }}$ mice was verified by mRNA quantification in the brain, comparing WT mice and nestin-Cre;EpoR $R^{W T / W T}$ mice, while its splenic expression as well as the hematocrit levels were unaffected (Supplemental Figure 3). For all subsequent studies with the nestin-Cre;EpoR ${ }^{\text {loxPlloxP }}$ mice, the nestin-Cre;EpoR ${ }^{W T / W T}$ mice were used as controls to counter the off-target effects of nestin promoter-driven Cre-recombinase expression and to ensure that the metabolic effects were seen due to lack of EpoR gene and not due to nestin-Cre transgene (33-35).

During DIO, male mice deficient for cerebral EpoR (e.g., nestin-Cre;EpoR ${ }^{\operatorname{lox} P / \text { loxP }}$ mice) gained significantly more weight than male controls (nestin-Cre;EpoR ${ }^{W T / W T}$ mice) (Figure $4 \mathrm{~A}$ ) and became more glucose intolerant after 4 weeks of HFD feeding (Figure 4B). Conversely, female mice lacking cerebral EpoR showed a moderate but significantly greater weight gain after only 2 weeks of HFD feeding and not at other time points tested (Figure 4C). Nevertheless, comparable to that in male mice, glucose tolerance was worse in female cerebral EpoR-deficient mice compared with female controls (Figure 4D). HFD-induced TNF- $\alpha$ expression in the MBH of male nestin-Cre;EpoR ${ }^{\operatorname{lox} P / l o x P}$ mice was also significantly higher than that in male nestin-Cre;EpoR ${ }^{W T / W T}$ mice (Figure 4, E and $\mathrm{F}$ ); however, Iba1 ${ }^{+}$microglial cells did not show any significant difference in cell numbers and fluorescence intensity between the 2 groups (Figure 4, E, G, and H). Activated microglial cells, stained with CD68 antibody, were more abundant in nestin-Cre;EpoR ${ }^{\text {loxP/loxP }}$ mice compared with nestin-Cre;EpoR ${ }^{W T / W T}$ mice (Figure 4, I and J). $\mathrm{CD}_{169^{+}}$peripheral myeloid cells were recruited more in the hypothalamus of nestin-Cre;EpoR ${ }^{\text {loxPlloxP }}$ mice than in nestin-Cre;EpoR ${ }^{W T / W T}$ mice, but P2Y12+ cells were not significantly different between the 2 groups (Figure $4, \mathrm{~K}$ and L). In summary, these studies show that, similar to EPO overexpression, endogenous physiological EPO/ EpoR signaling in the brain plays an important role in preventing HFD-induced weight gain and inflammation in male mice and can regulate glucose tolerance in both male and female mice.

Male Tg21 mice are protected from pituitary-adrenal activation during DIO. The hypothalamus-pituitary-adrenal (HPA) axis is a critical neuroendocrine control over stress response induced during DIO. The hypothalamus and pituitary gland function to maintain energy expenditure. Corticotropin releasing factor (CRF) released from the hypothalamus induces ACTH secretion from the pituitary, which in turn drives release of corticosterone from the adrenal gland. The ACTH level during NCD showed a small reduction in $\mathrm{Tg} 21$ mice, which increased in both WT and Tg21 mice during DIO to become 1.7-fold greater in WT mice compared with $\mathrm{Tg} 21$ mice (Figure 5A). Corticosterone levels were similar in WT and Tg21 mice during NCD and increased only in WT mice during DIO, becoming 2-fold greater (Figure 5B). The inflammatory marker C-reactive protein was higher in WT mice than in Tg21 mice during NCD, was induced in DIO in both WT and Tg21 mice, and remained significantly higher in WT mice (Figure 5C). This observation, too, was sex specific; in female WT and Tg21 mice no differences were observed in serum levels of ACTH, corticosterone, and C-reactive protein during NCD or HFD (data not shown).

Fgf21 and adiponectin levels during DIO. Female mice appear to be protected from hypothalamus inflammation and pituitary-adrenal activation, but, similar to male mice, they exhibit glucose intolerance during DIO. Fgf21, a cytokine produced primarily in liver, is involved in regulation of glucose metabolism and insulin sensitivity, parameters that are improved in male and female $\mathrm{Tg} 21$ mice during NCD and DIO. During NCD, Fgf21 was elevated in male and female $T g 21$ mice. However, after 1 and 3 months on HFD, Fgf21 levels increased in male and female WT mice beyond the levels in $T g 21$ mice, which remained unchanged (Figure 5, D and E). Adipocytes are one main target of Fgf21 where Fgf 21 promotes adiponectin production. During NCD, male and female $\operatorname{Tg} 21$ mice showed a significantly higher adiponectin level, although fat mass was comparable to that of WT mice (Figure 5, F and G, and Table 1). Adiponectin levels increased during HFD feeding in both Tg21 and WT mice, but there 

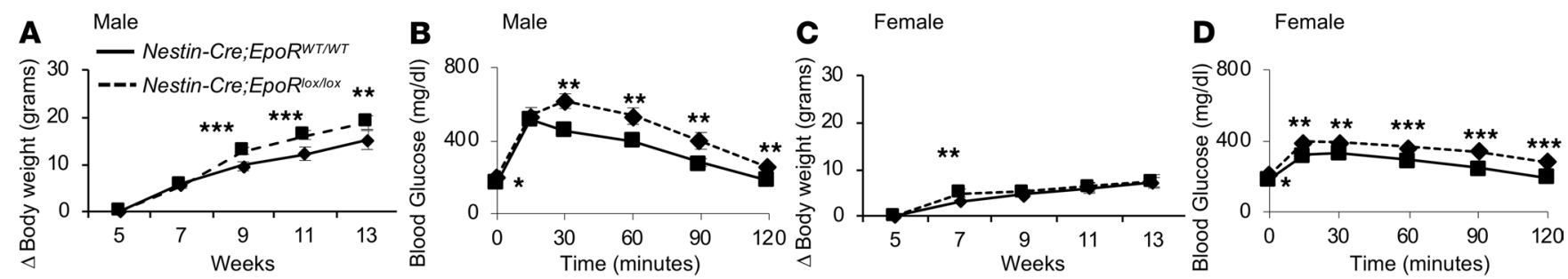

E
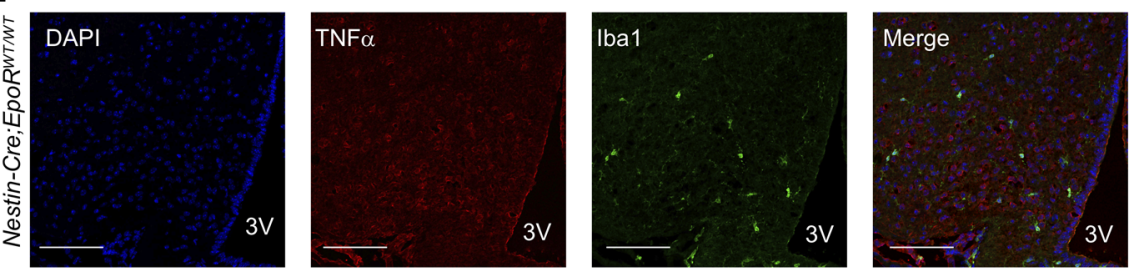

F
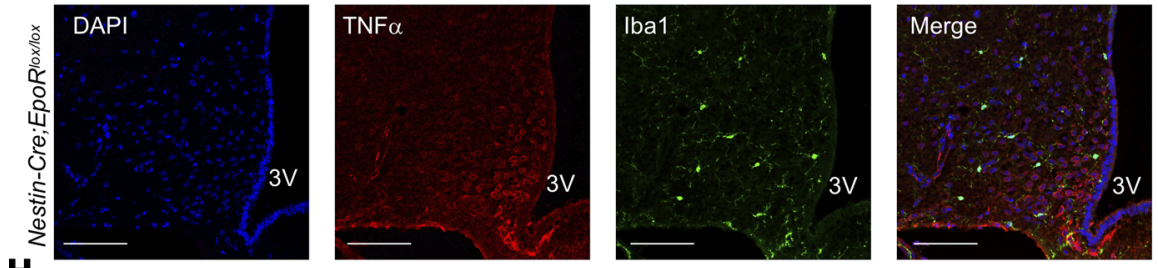

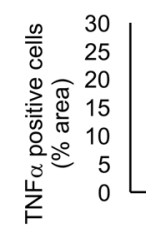
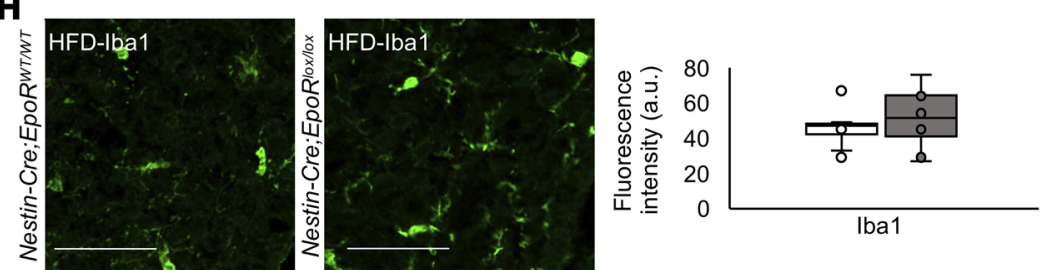

Nestin-Cre;EpoR ${ }^{\text {WTWT }}$

Nestin-Cre;EpoR Rox/10x

\section{I}
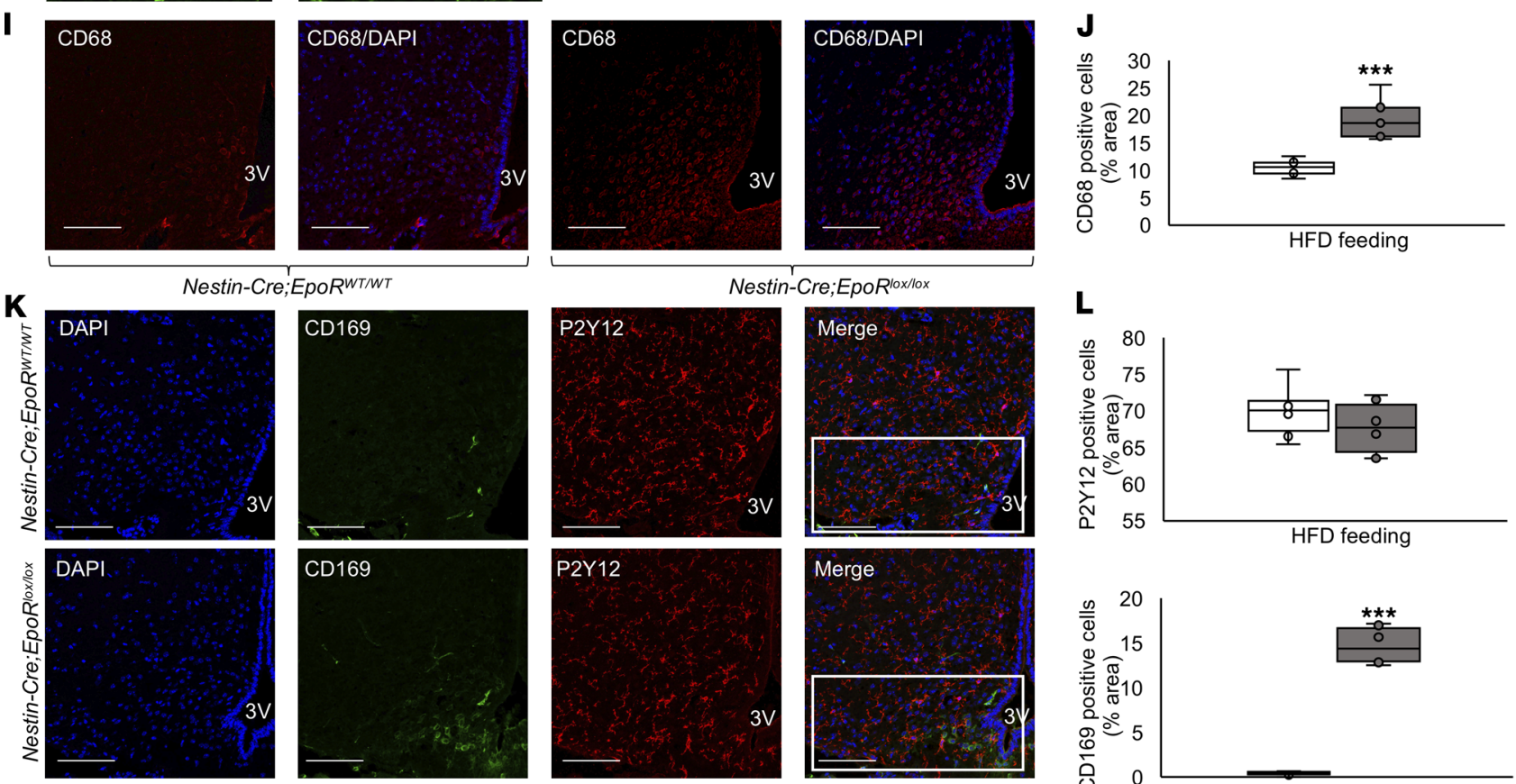

Nestin-Cre;EpoR ${ }^{\text {WT/W }}$
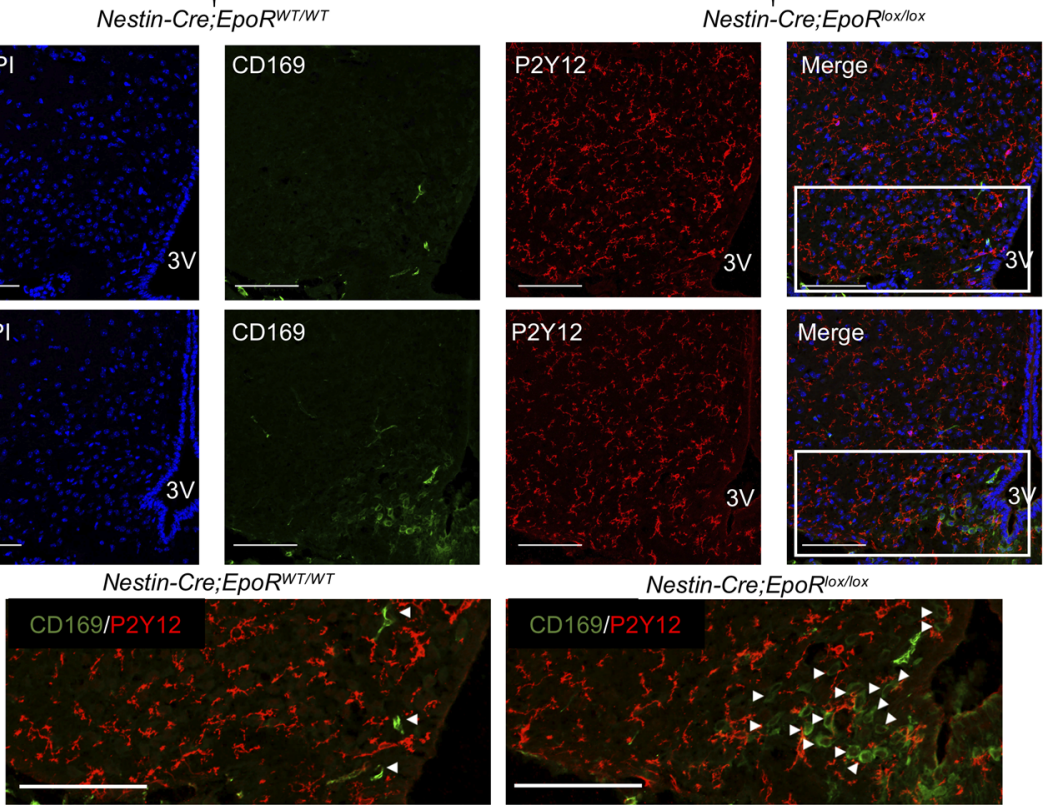

Nestin-Cre:EpoR/ox/lox
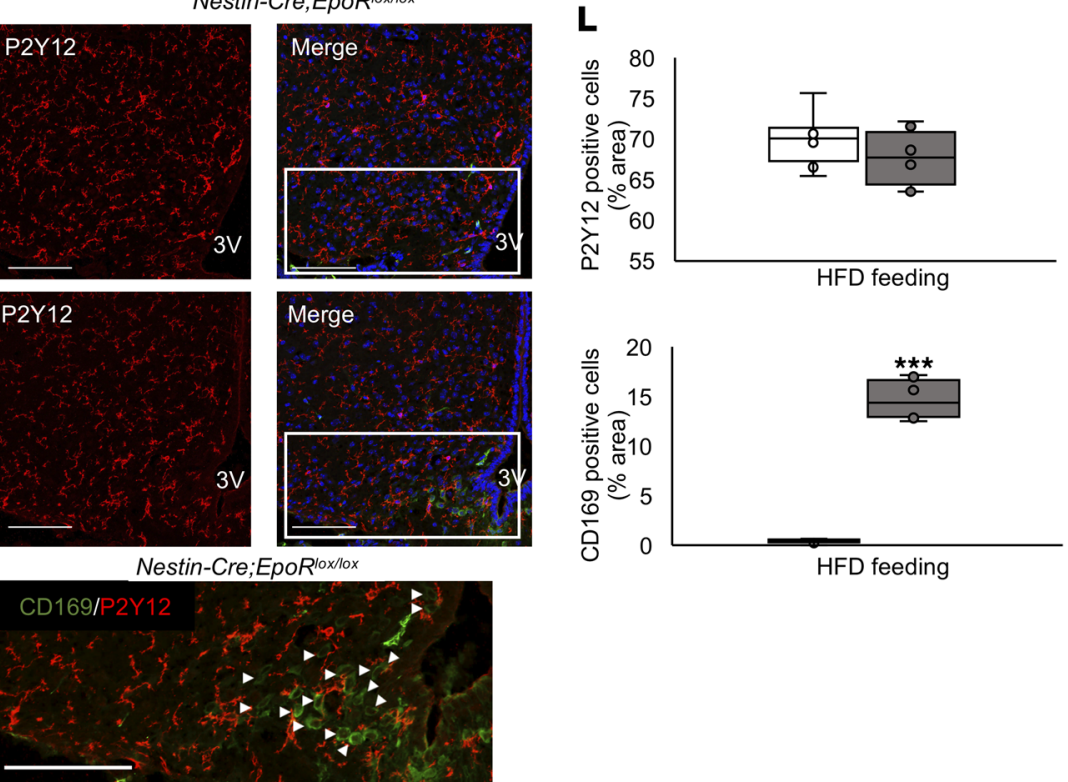

HFD feeding 
Figure 4. Knockout of the EpoR gene in neuronal cells in mice increased weight gain, glucose intolerance, hypothalamus inflammation, and myeloid cell recruitment in the MBH. (A and C) Weight gain during HFD feeding from 5 to 13 weeks of age. (B and $\mathbf{D})$ Glucose tolerance test was carried out after 4 weeks of HFD feeding at age 9 weeks. (E-H) Representative hypothalamic sections of age-matched (8 weeks) male nestin-Cre;EpoR ${ }^{\text {WT/WT }}$ and nestin-Cre; EpoR $R^{\text {IoxP/loxP }}$ mice after 3 weeks of HFD feeding stained for the nuclear marker, DAPI; inflammatory marker, TNF- $\alpha$; microglial cell marker, Iba1 (E). (F and G) Quantification was done for TNF- $\alpha^{+}$and Ibal1+ cells. (H) Representative fields of view in HFD-fed male nestin-Cre;EpoR ${ }^{W T / W T}$ and nestin-Cre;EpoR ${ }^{\text {loxP/loxP }}$ mice, analyzed for quantification of fluorescence intensity of Iba1. (I-J) Male nestin-Cre;EpoR ${ }^{W T / W T}$ and nestin-Cre;EpoR $R^{\text {loxP } / 10 \times P}$ mice after 3 weeks of HFD feeding were also stained for activated microglial cell markers, CD68 and DAPI, in the MBH, and (I) representative images are shown. (J) Quantification was done for CD68+ cells. (K and L) Representative images from CD169 and P2Y12 staining of hypothalamic sections from male nestin-Cre;EpoR ${ }^{W T / W T}$ and nestin-Cre;EpoR ${ }^{\text {loxP/loxP }}$ mice after 3 weeks of HFD feeding, and visualization of boxed areas without DAPI staining and arrowheads pointing to CD169+ cells (K). Quantitative analysis results of P2Y12+ and CD169+ cells (L). Scale bar: $20 \mu \mathrm{m}$ (H); $100 \mu \mathrm{m}$ (E, I, and K). Original magnification, $\times 40$ (except in $\mathbf{H}, \times 80$ ). $3 \mathrm{~V}$, third ventricle. Each image is representative of $n=8-10 /$ group. For line graph, each data point represents mean \pm SD. In box-and-whisker plots, bounds denote the 25 th to 75 th percentile, the lines represent the medians, and whiskers indicate the range from minimum to maximum values and includes outliers. ${ }^{*} P<0.05$, ${ }^{* *} P<0.01,{ }^{* *} P<0.001$ (2-way ANOVA).

was a higher elevation in male and female $T g 21$ mice. This observation was surprising, as male WT mice had more fat mass (Figure $5 \mathrm{~F}$ and Table 1); it suggests that during DIO, despite higher Fgf2 1 in WT mice (Figure 5, D and E), adiponectin levels were not concomitantly higher in these mice. On the other hand, $\mathrm{Tg} 21$ male mice and female mice showed a greater sensitivity to Fgf21, as reflected in adiponectin levels (Figure 5, F and G, and Table 1).

BKlotho expression in WAT and Fgf21 signaling in hypothalamus during DIO. While systemic metabolic effects of Fgf21 are mediated mainly by adiponectin from WAT (36), hepatic Fgf21 can regulate glycogen synthesis and ketone body production $(37,38)$. Fgf21 can also cross the blood-brain barrier and act as a messenger between the liver and hypothalamus, thereby regulating CRF expression and adrenal corticosterone levels (39). In the presence of the critical coreceptor $\beta$ Klotho, Fgf21 binds to isoforms of Fgf receptor 1, 2, 3, and 4, leading to dimerization and autophosphorylation of the Fgf receptor, followed by downstream activation of Erk1/2 that translocates and activates specific transcription factors in the nucleus. Expression of Fgf receptors $1,2,3$, and 4 was unchanged during NCD and after 3 months of HFD (data not shown), while $\beta$ Klotho expression in WAT during DIO was significantly reduced in male and female WT mice in contrast to male and female $T g 21$ mice, which showed no difference in $\beta K$ Klotho expression in WAT, liver, and hypothalamus during NCD or HFD (Figure 6, A-C). During DIO, Fgf21 in serum was higher in male and female WT mice (Figure 5, D and E), and on the other side of the blood-brain barrier, we detected higher $C R F$ expression in the hypothalamus in male WT mice compared with that in $T g 21$ mice but $C R F$ expression was comparable in female WT and Tg21 mice (Figure 6, D and E). This suggested that, while Fgf21 increased in WT mice during DIO, it did not lead to elevated adiponectin secretion, possibly due to $\beta$ Klotho downregulation in WT WAT compared with that in $\mathrm{Tg} 21$ mice. Moreover, CRF secretion from the hypothalamus was higher in male WT mice compared with male $T g 21$ mice but remained unchanged in female mice.

Intracerebroventricular EPO administration during DIO prevents weight gain, lowers blood glucose levels, and attenuates hypothalamic inflammation. To determine whether administration of recombinant human EPO in brain is protective for DIO, $10 \mathrm{U} / \mathrm{d}$ EPO was infused continuously for 2 weeks into the lateral cerebral ventricle of male mice, together with HFD feeding. Saline infusion was used as a control. EPO infusion reduced cumulative weight gain and fat mass from days 7 through 14 of HFD feeding, with no significant effect on in lean mass (Figure 7, A-C). EPO brain infusion resulted in a modest increase in hematocrit at week 1 and a modest decrease at week 2 compared with saline-infused mice (Figure 7D). The week 2 hematocrit level in EPO-infused mice was significantly lower than the week 1 level, which could possibly be due to a feedback from the brain through the sympathetic nervous system (40). Fasting glucose levels were significantly lower in male mice receiving EPO brain infusion (Figure 7E). Finally, at the end of 2 weeks of EPO infusion, hypothalamic expression of inflammatory markers $I L-1 \alpha, I L-6, S O C S 3$, and TNF- $\alpha$ was down regulated while expression of antiinflammatory marker $I L-10$ was increased (Figure $7 F$ ). These findings suggest that pharmacological administration of EPO into the brain during HFD feeding can prevent weight gain and hypothalamus inflammation and improve glucose tolerance.

EPO action in female Tg21 mice is blunted by estrogen's protective effect against DIO. Male mice are more susceptible to DIO than female mice (41), and obesity-related hypothalamic inflammation affects male but not female rodents (42-44). Similarly, microglial cell response during HFD feeding is sexually dimorphic, with male mice presenting more accumulation and activation of microglial cells in MBH (42). Moreover, the metabolic response of EPO protection from DIO is sex specific and is observed predominantly in male mice (18). Here, we found that inflammatory stress in female WT and Tg21 hypothalamus was comparable during 
A

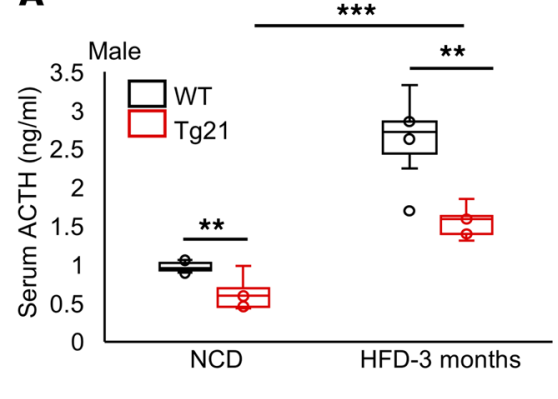

B

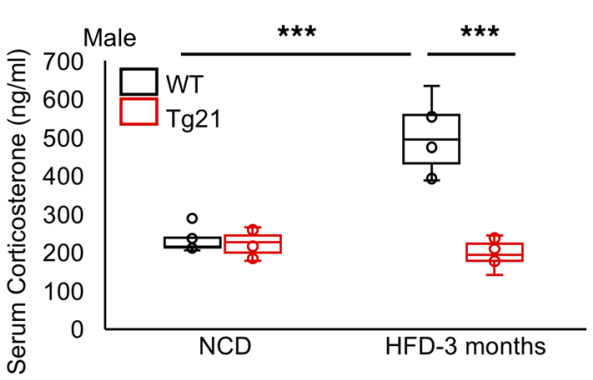

C

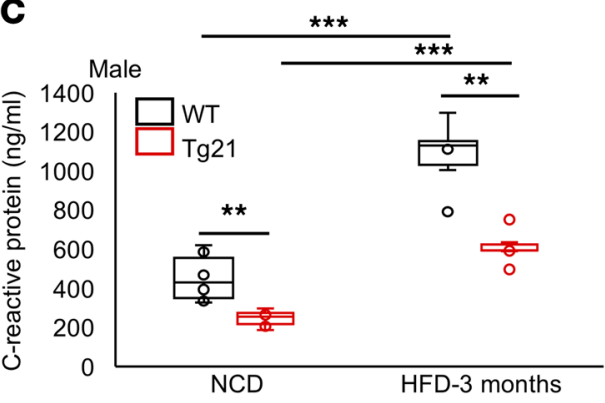

D

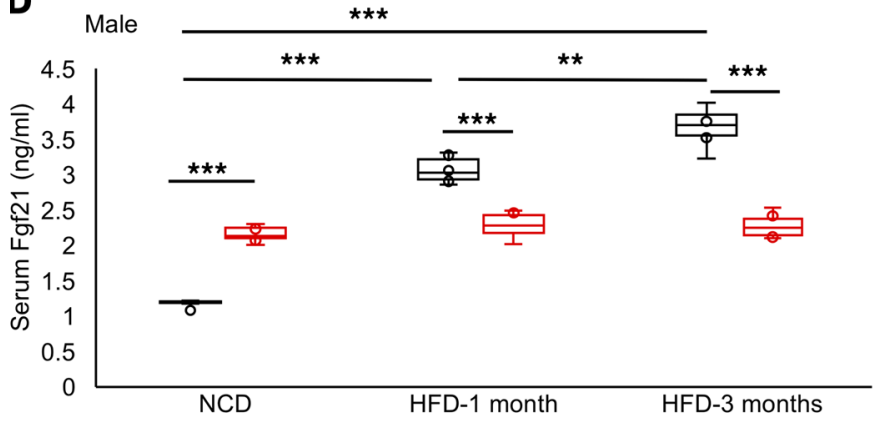

E

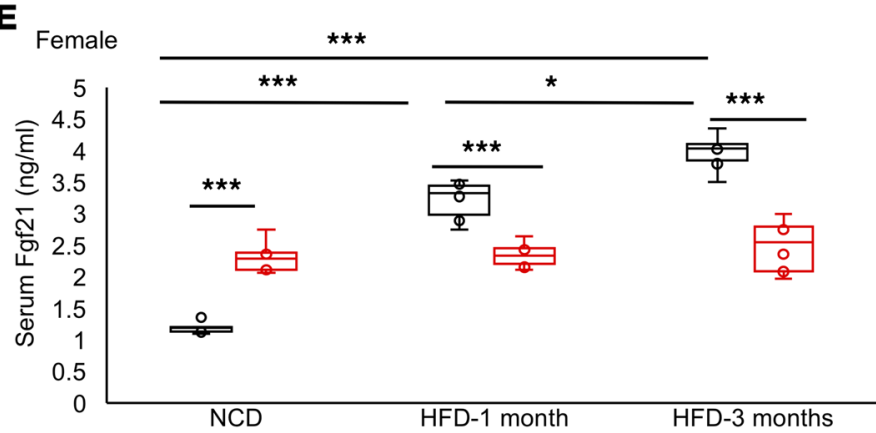

$\mathbf{F}$

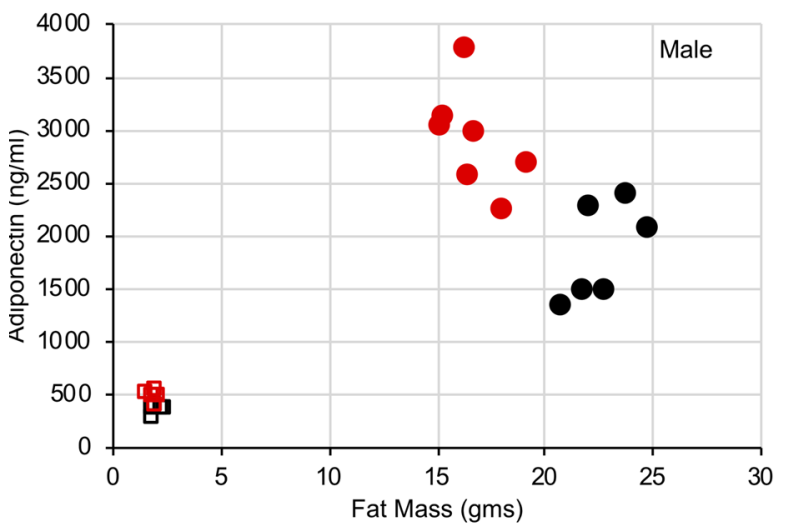

$\square W T$ mice- NCD

$\square$ Tg21 mice- NCD
G

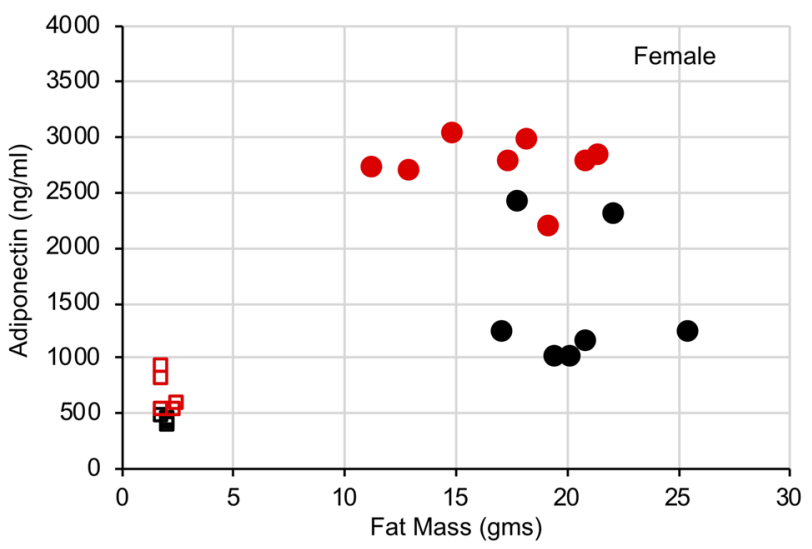

WT mice- 3 months HFD

Tg21 mice- 3 months HFD

Figure 5. Tg21 mice had lower serum levels of ACTH, corticosterone, C-reactive protein, and Fgf21 but higher adiponectin levels during development of DIO. (A-C) Serum levels of ACTH (A), corticosterone (B), and C-reactive protein (C) in age-matched (5 months) male Tg21 and WT mice during NCD or after 3 months of HFD feeding. (D and E) Serum levels of Fgf21 in male (D) and female (E) age-matched (5 months) Tg21 and WT mice during NCD or after 1 month or 3 months of HFD feeding. (F and $\mathbf{G}$ ) Fat mass versus serum adiponectin levels in male (F) and female (G) agematched (5 months) Tg21 and WT mice during NCD or after 3 months of HFD feeding. In box-and-whisker plots, bounds denote the 25 th to 75 th percentile, the lines represent the medians, and whiskers indicate the range from minimum to maximum values and includes outliers. ${ }^{*} P<0.05$, ${ }^{* *} P<0.01,{ }^{* * *} P<0.001$ (2-way ANOVA), $n=6$-8/group.

HFD feeding and that EPO did not elicit an additional protective effect against DIO in female mice (Supplemental Figures 1 and 2). To assess the protective effect of estrogen in DIO, we used ovariectomized (OVX) and sham controlled female mice undergoing a 6-week HFD feeding period. Body weight gain and fat mass after 3 and 6 weeks were greatest in OVX WT mice (Figure 8, A and B), suggesting that estrogen plays a dominant role in preventing DIO in female mice and estrogen reduction in OVX mice results in significantly higher body weight and fat mass gain during HFD feeding. Furthermore, EPO overexpression in the brain protects against DIO in OVX Tg21 mice with loss of estrogen protection compared with OVX WT mice.

Assessment of direct inflammatory effects in MBH of WT and $T g 21$ mice subjected to ovariectomy and HFD feeding showed that reduction of estrogen production in OVX WT mice increased TNF- $\alpha^{+}$cells 
Table 1. Fat mass and serum adiponectin levels in male and female WT and Tg21 mice

\begin{tabular}{lccccccc}
\hline & & Male WT mice & Male Tg21 mice & P value & Female WT mice & Female Tg21 mice & P value \\
NCD & Fat mass $(\mathrm{g})$ & $2.21 \pm 0.26$ & $2.00 \pm 0.21$ & 0.192 & $2.05 \pm 0.13$ & $2.06 \pm 0.34$ & 0.976 \\
NCD & Adiponectin $(\mathrm{ng} / \mathrm{ml})$ & $363.44 \pm 41.82$ & $495.58 \pm 49.62$ & $\mathbf{0 . 0 0 2}$ & $423.73 \pm 32.45$ & $659.11 \pm 176.13$ & $\mathbf{0 . 0 3 5}$ \\
HFD & Fat mass $(\mathrm{g})$ & $22.71 \pm 1.47$ & $16.8 \pm 1.48$ & $\mathbf{1 . 7 2} \times \mathbf{1 0}-\mathbf{5}$ & $20.42 \pm 2.81$ & $17.01 \pm 3.69$ & $\mathbf{0 . 0 0 0 1}$ \\
HFD & Adiponectin $(\mathrm{ng} / \mathrm{ml})$ & $1851.78 \pm 455.79$ & $2926.15 \pm 481.02$ & $\mathbf{0 . 0 0 2}$ & $1477.05 \pm 603.38$ & $2742.66 \pm 255.37$ & 0.067
\end{tabular}

Fat mass and serum adiponectin levels in male and female age-matched ( 5 months) Tg21 and WT mice during NCD or after 3 months of HFD feeding ( $n=6$-8/group) (mean \pm SD). Statistical significance was tested by 2 -way ANOVA, and significant $P$ values are shown in bold.

and $\mathrm{Iba}^{+}$cells in hypothalamic sections, indicating an increase in inflammatory response and microglial cell number compared with sham WT mice (Figure 8, C and D). In contrast, OVX Tg21 mice with elevated brain EPO did not show any such increase compared with sham $T g 21$ mice (Figure 8, C and D). Female sham WT and sham Tg21 mice did not show any difference in TNF- $\alpha$ or Iba1 staining. These findings suggested that estrogen protection for DIO includes concomitant reduced hypothalamic inflammation and that loss of estrogen production in OVX female mice with HFD feeding increased hypothalamic inflammation. However, female OVX Tg21 mice with elevated brain EPO did not exhibit a hypothalamus inflammatory response during HFD feeding due to the EPO antiinflammatory effect that was also seen in $T g 21$ male mice with elevated brain EPO (Figure 2) and after intracerebroventricular EPO administration (Figure 7).

\section{Discussion}

The hypothalamus plays a master role in coordinating food intake and energy expenditure, and hormones, such as leptin and insulin, act as messengers between the periphery and hypothalamus. These hormones inform about peripheral energy stocks and trigger physiological responses in hypothalamus, controlling hunger, glucose metabolism, and thermogenesis. We previously found that EPO treatment in WT mice decreases body weight and fat mass by increasing energy expenditure $(12,15)$ and can directly activate POMC expression by inducing the STAT3 signaling pathways after subcutaneous administration of recombinant human EPO at $3000 \mathrm{U} / \mathrm{kg}$ body weight dose (13). Similarly, intraperitoneal administration of EPO at $5000 \mathrm{U} / \mathrm{kg}$ body weight has been shown to be neuroprotective in rodent models of focal brain ischemia (45) and schizophrenia (46). These studies show that EPO administered at such doses can cross the bloodbrain barrier and elicit neuroprotective as well as metabolic effects. Similarly, endogenous physiological $\mathrm{EPO} / \mathrm{EpoR}$ signaling also regulates metabolism, as mice lacking the EpoR gene in nonhematopoietic tissues exhibit fat gain, glucose intolerance, and insulin resistance, resulting from low energy expenditure that is primarily due to loss of endogenous EPO response in WAT and hypothalamus (12). These mice presented with high plasma ACTH concentration due to higher ACTH secretion from anterior pituitary (14). These nonerythropoietic EPO activities were attributed to EPO response in multiple tissues, including the brain. Here, we focused on elucidating EPO's brain-specific role that may contribute to the metabolic response, especially during DIO. As a model with elevated brain EPO without increase in EPO-stimulated erythropoiesis, we used Tg21 mice overexpressing EPO in the brain and WT mice with intracerebral ventricular EPO pumps. Increased cerebral EPO levels prevented weight and fat gain during HFD feeding in male mice, and male $T g 21$ mice exhibited higher energy expenditure. Absence of EpoR in the brain and other nonhematopoietic cells led to reduced locomotor activity and energy expenditure, while EPO treatment in WT mice increased these parameters (12). Furthermore, elevated brain EPO in Tg21 mice or in mice administered high-dose EPO increased maximal aerobic capacity and time to exhaustion during exercise (47), underlining the widespread effect of cerebral EPO on physical energy expenditure. Intracerebroventricular administration of EPO has been previously shown to be protective against cortical infarction, loss of thalamic neurons, behavioral deficits, and reperfusion injury in a rodent model of permanent middle cerebral artery occlusion (48-50). Here, our findings show that such continuous EPO infusion can also be protective against DIO and related glucose intolerance and hypothalamic inflammation. We detected a small but significant decrease in hematocrit in EPO-infused mice at week 2, which could possibly be due to feedback regulation mediated by the high EPO levels in the brain (40). Previous studies have shown that the sympathetic nervous system and its constituents, the sympathetic nerve fibers and neural crest-derived cells, are critical regulators of hematopoietic stem cell behavior during homeostasis and stress (51-53). 
A
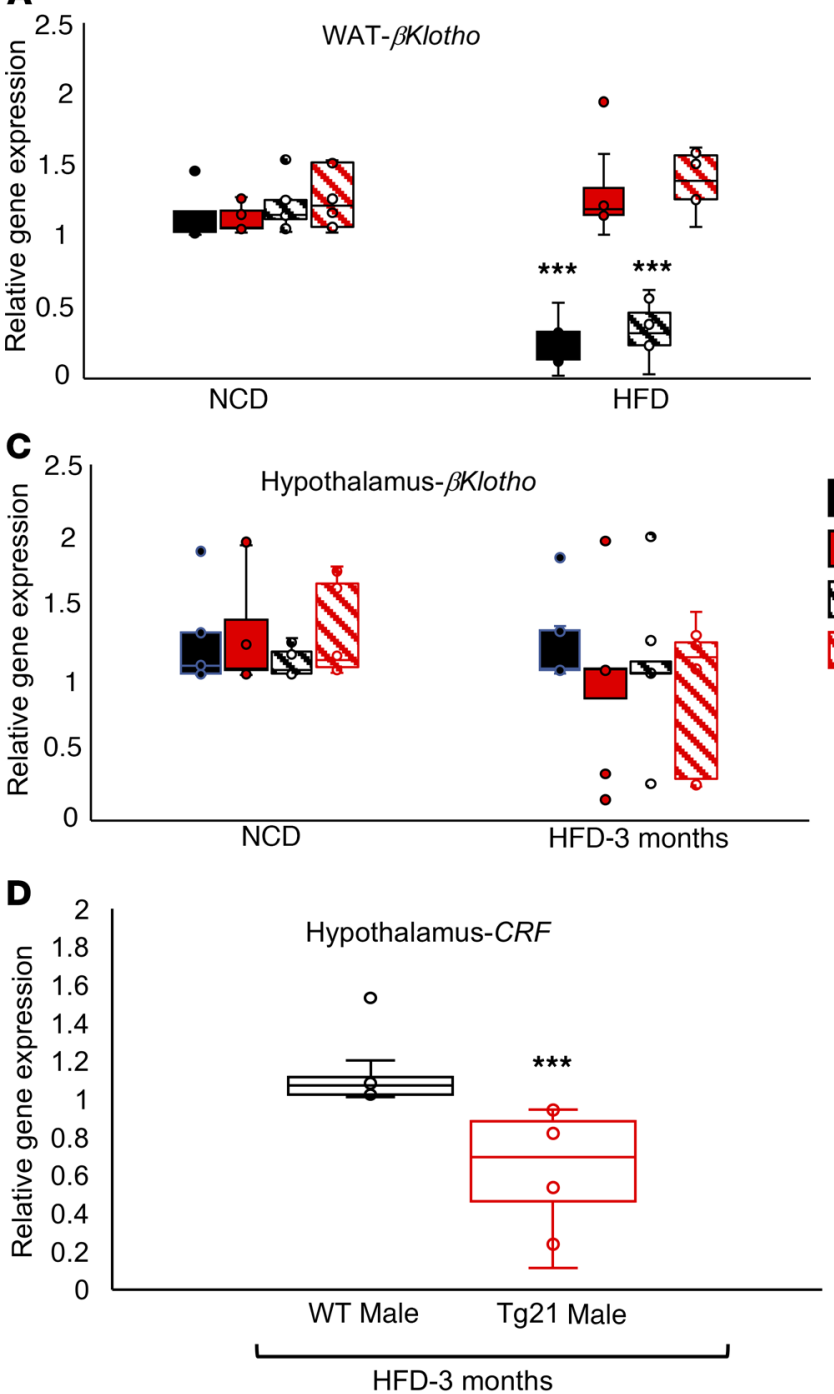

B

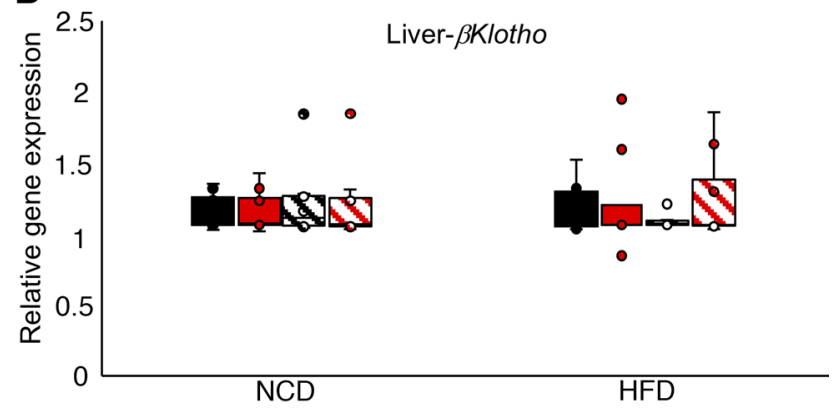

WT male

Tg21 male

WT female

Tg21 female

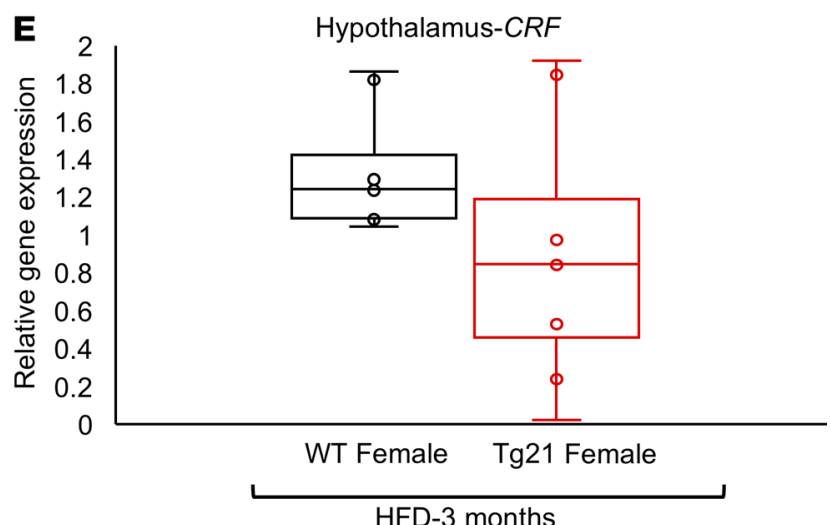

HFD-3 months

Figure 6. During DIO, Fgf21 coreceptor $\beta$ Klotho mRNA expression was reduced in WAT of male and female WT mice while Fgf21 signaling was activated in hypothalamus of male WT mice. (A-C) Quantitative mRNA measurements of $\beta$ Klotho in WAT (A), liver (B), and hypothalamus (C) in age-matched (5 months) male and female Tg21 and WT mice during NCD or after 3 months of HFD feeding. Gene expression is presented as relative to male or female WT mice on NCD and adjusted for Gapdh mRNA expression. (D and E) Gene expression of CRF in the hypothalamus of male (D) and female (E) Tg21 and WT mice (age 5 months) after 3 months of HFD feeding determined by quantitative RT-PCR, normalized to WT control and adjusted to Gapdh gene expression ( $n=6-8$ /group). In box-and-whisker plots, bounds denote the 25 th to 75 th percentile, the lines represent the medians, and whiskers indicate the range from minimum to maximum values and includes outliers. ${ }^{* *} P<0.001$ (2-way ANOVA).

We observed that, in male $T g 21$ mice, reduced body weight gain and fat mass accumulation was accompanied by improved glucose metabolism and insulin sensitivity during both NCD and HFD feeding. Interestingly, improved glycemic control in $\mathrm{Tg} 21$ mice was seen in both male and female mice, possibly regulated by Fgf 21 and adiponectin action. Fgf 21 is a hormone made primarily in the liver and acts as a key metabolic regulator, essential for maintaining glucose homeostasis (54). In mice, Fgf21 is induced at the transcriptional level and released from liver in response to nutritional stresses, such as HFD feeding (55). Similarly, in humans, circulating FGF21 levels correlate positively with BMI (56), and plasma FGF21 is elevated in obese and diabetic humans (57). This suggests that during obesity Fgf21 fails to exert its expected effects on glucose homeostasis, which is affected by several factors, including decreased expression of Fgf21 coreceptor (58). Our findings show that increased Fgf21 during DIO in WT mice was accompanied by lower adiponectin production from WAT, possibly due to downregulation of the Fgf 21 coreceptor $\beta$ Klotho. Adiponectin, a major downstream effector of Fgf21, contributes to the metabolic benefits of Fgf21 in both liver and skeletal muscles (36). Although 
A
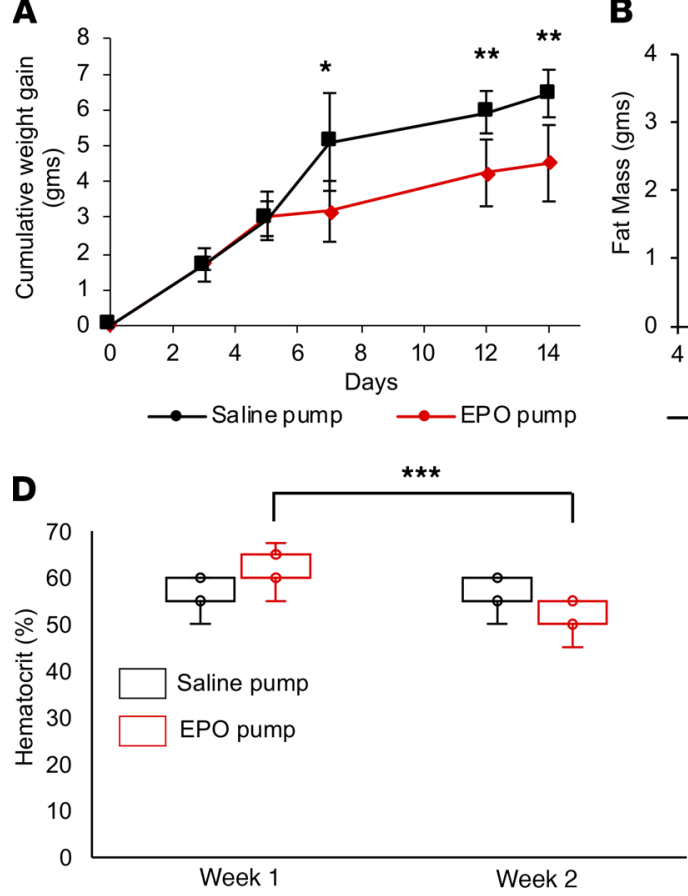

$\mathbf{F}$

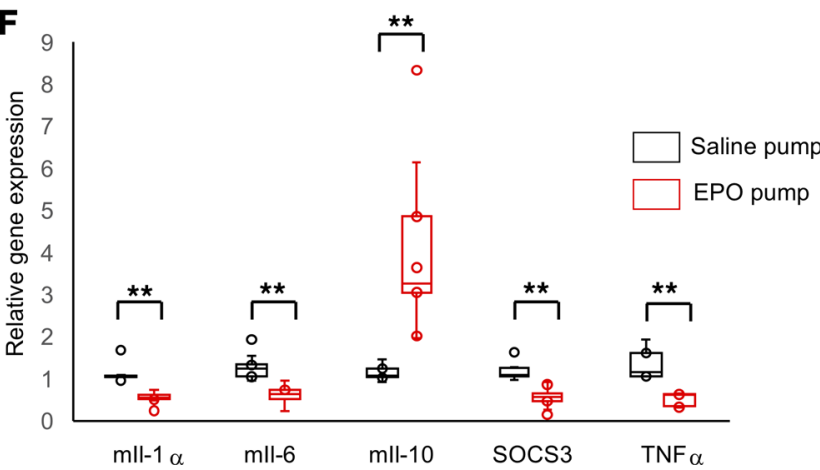

B

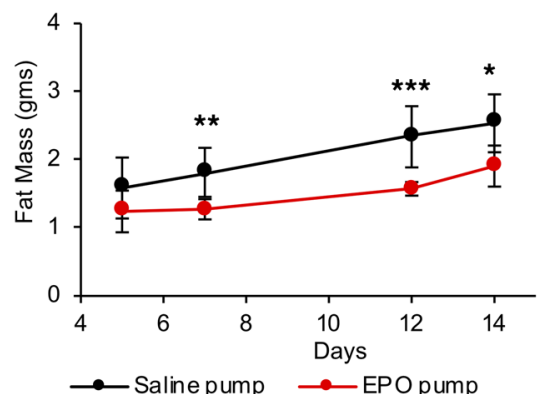

E

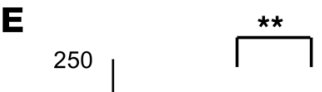

C

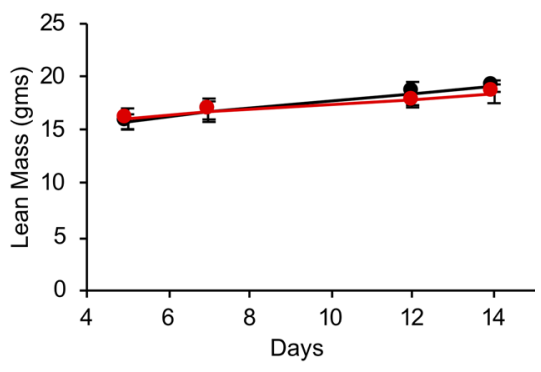

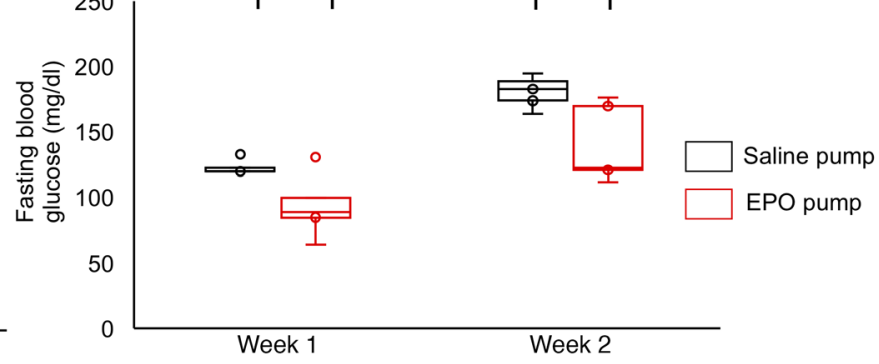

Figure 7. Intracerebroventricular administration of EPO protected mice from DIO and reduced fasting blood glucose and inflammation, without affecting hematocrit. (A-C) Mice at age 5 weeks were infused with recombinant human EPO or saline and fed HFD for 2 weeks. Cumulative weight gain (A), fat mass (B), and lean mass (C) were measured at indicated time points. ( $\mathbf{D}$ and $\mathbf{E})$ At the end of weeks 1 and 2, hematocrit (D) and blood glucose (E) were measured for EPO- or saline-infused mice. (F) Gene expression of inflammatory markers in the hypothalamus at the end of 2 weeks of EPO-infused mice, as determined by quantitative RT-PCR, normalized to saline control and adjusted to Gapdh gene expression. In box-and-whisker plots, bounds denote the 25th to 75th percentile, the lines represent the medians, and whiskers indicate the range from minimum to maximum values and includes outliers. ${ }^{*} P<0.05$, ${ }^{* *} P<0.01$, ${ }^{* * *} P<0.001$ (2-way ANOVA), $n=6$-8/group.

this effect is seen in both male and female mice, the hypothalamus response of Fgf2 1 with respect to $C R F$ expression showed sexual dimorphism without any difference in Fgf21 receptor and $\beta$ Klotho expression. Higher $C R F$ expression in the hypothalamus of male WT mice (Figure 6D) could ultimately cause higher ACTH and corticosterone levels in the serum (Figure 5, A and B). Pharmacologic studies have also revealed that Fgf21 results in elevated blood corticosterone in mice (59).

Excess consumption of HFD results in hypothalamus inflammation analogous to other peripheral tissues, including WAT, liver, and skeletal muscles (26). Hypothalamic inflammation is one of the major pathways by which overnutrition can cause obesity. Disruption of inflammatory molecules has been shown to protect against DIO. For example, ablation of IKK $\beta$ in AgRP neurons (60), knockout of MyD88 in the CNS (61), or inhibition of TLR4 and TNF- $\alpha$ in the hypothalamus (62) produce beneficial metabolic effects in HFD-fed mice. The inflammatory effect is accompanied by infiltration by bone marrow-derived $\mathrm{CD} 169^{+}$myeloid cells that is critical for causing "microgliosis" in MBH and metabolic dysfunction (63). To disrupt the EPO response in the brain, we targeted deletion of EpoR in nestin ${ }^{+}$cells, including their progeny, that includes neurons but not microglial cells $(64,65)$. Our attempts at EpoR gene knockout in all cell types by viral Cre administration in $E p o R^{\text {loxPlloxP }}$ mice resulted in nonspecific recombinase action, even in WT mice. Therefore, the effects seen in nestin-Cre:EpoR ${ }^{\text {loxPloxP }}$ mice could only be contributed by physiological EPO function in neuronal cells. Our findings in male $T g 21$ mice and in male WT mice with intracerebroventricular EPO infusion directly link EPO protection and lower hypothalamus inflammation. In mouse WAT, peripheral EPO treatment during DIO prevents WAT inflammation, reduces macrophage infiltration, and improves glucose tolerance and insulin sensitivity 

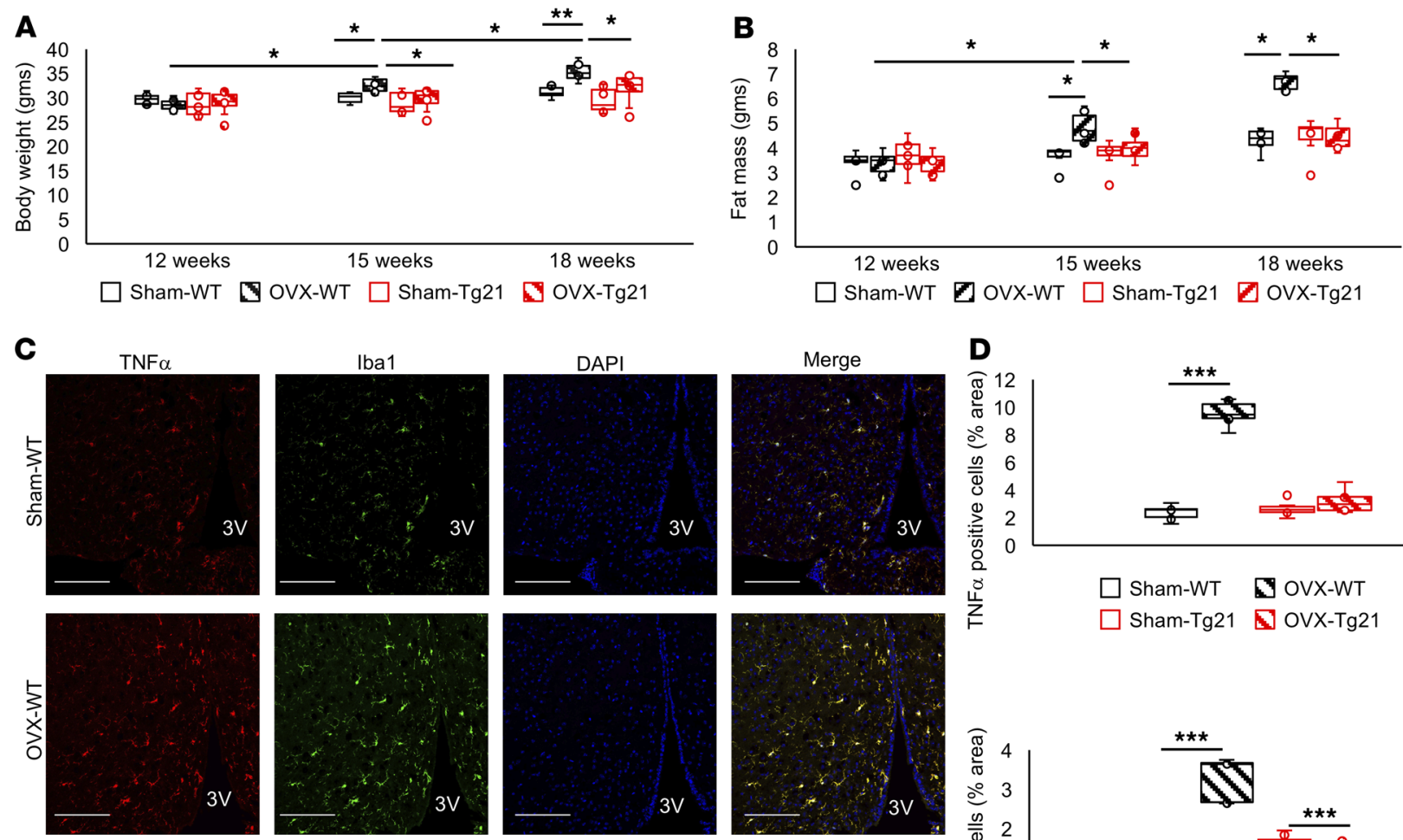

4
2
0
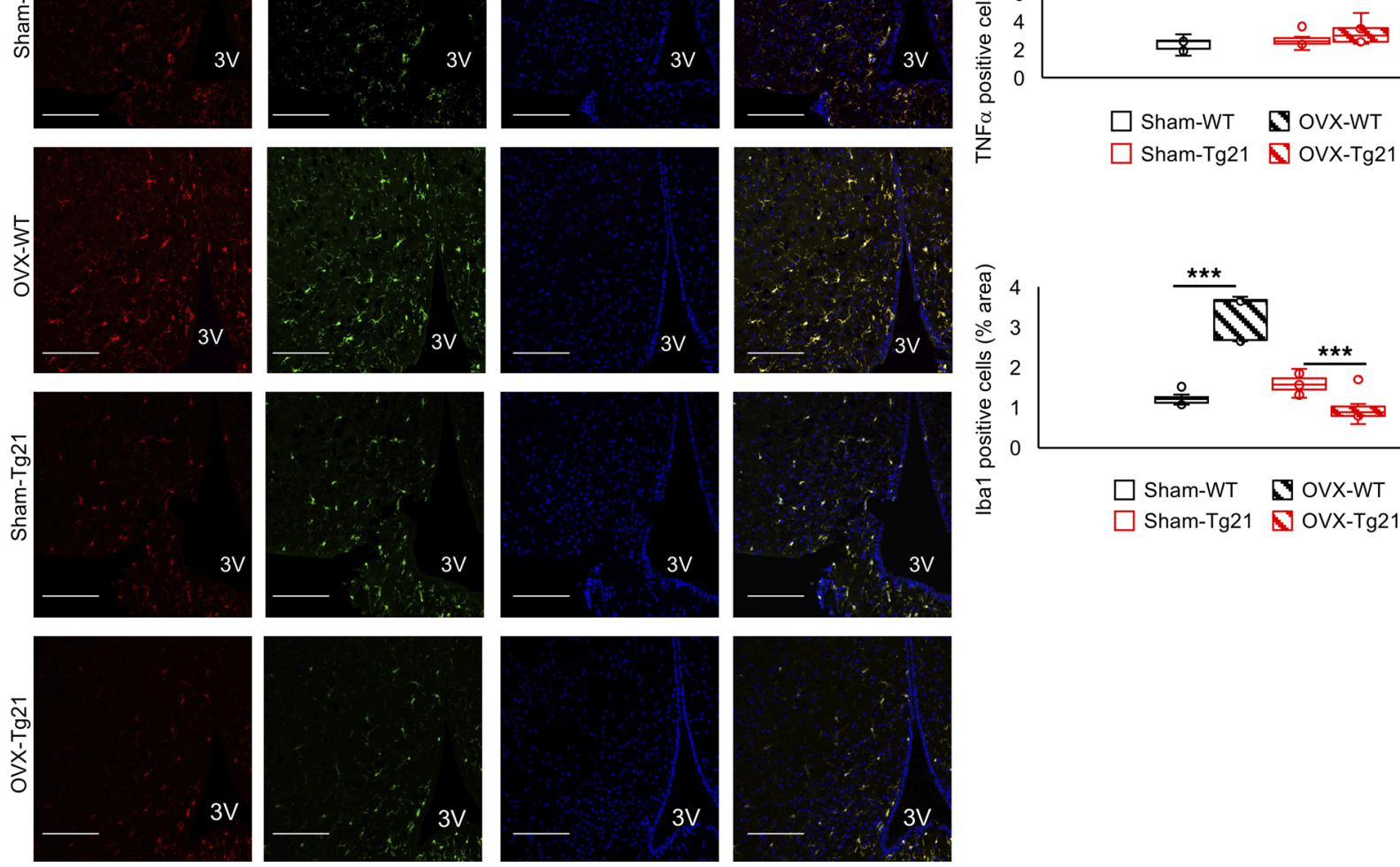

Figure 8. Ovariectomized WT mice but not Tg21 mice lose the protective effect against DIO. (A and B) Female WT and Tg21 mice underwent ovariectomy (OVX) or sham surgery (Sham) at 12 weeks of age and were fed HFD for the next 6 weeks. Body weight (A) and fat mass (B) were measured every 3 weeks. (C) Representative hypothalamic sections after 6 weeks of HFD stained for the nuclear marker, DAPI; inflammatory marker, TNF- $\alpha$; and activated microglial cell marker, Iba1, in MBH of sham or ovariectomized Tg21 and WT mice. Scale bar: $100 \mu \mathrm{m}$. Original magnification, $\times 40.3 \mathrm{~V}$, third ventricle. (D) Quantitative analysis to show TNF- $\alpha^{+}$and Iba1 ${ }^{+}$cells. Each image is representative of $n=6$-8/group. In box-and-whisker plots, bounds denote the 25 th to 75th percentile, the lines represent the medians, and whiskers indicate the range from minimum to maximum values and includes outliers. ${ }^{*} P<0.05,{ }^{* *} P$ $<0.01,{ }^{* *} P<0.001$ (2-way ANOVA).

(6). Male $\operatorname{Tg} 21$ mice had reduced levels of the inflammatory marker C-reactive protein in serum, during both NCD and HFD feeding, suggesting that peripheral inflammation is also lower in $T g 21$ mice independent of weight and fat mass difference.

EPO regulation of body weight and fat mass in female mice during HFD feeding is minimal but is readily apparent in OVX mice and is masked in OVX mice with estradiol supplementation (18). Our present studies were carried out in randomly cycling females, as random cycling better resembles a chronic condition, such as DIO, in cycling females. In addition, previous studies done in cycling female mice have not found any significant difference in inflammatory cytokines throughout the estrous cycle (66), and the HPA axis response to endotoxins does not vary with different stages of the normal estrous cycle (67). Moreover, female mice have been shown to be resistant to hypothalamic inflammation and DIO through estrogen effects, and ovariectomy can eliminate these protective effects (41-43). Results from female $T g 21$ 
mice also suggest that estrogen can elicit the maximal protective effect against hypothalamic inflammation and DIO and that EPO overexpression in brain is not additionally beneficial in female mice. However, loss of estrogen as occurring in OVX WT mice results in increased hypothalamic inflammation on HFD feeding, and the protective effect of elevated brain EPO for hypothalamic inflammation in DIO is demonstrated in OVX Tg21 mice. An effect of estrogen on EPO-regulated functions in the brain and carotid bodies has been already postulated in the past using the same and similar EPO-overexpressing mouse models $(68,69)$. It was shown that cerebral EPO mediates the neuronal control of the ventilatory response to hypoxia in a sex-dependent manner and that estradiol interferes with EPO's effect on this response (70). Taken together, all these findings strongly support the evidence that EPO and estrogen may be functioning through similar mechanisms regulating metabolism and ventilation both in the brain and in the periphery.

Evidence of decreased expression of EPO during aging may be relevant to the development of neuroinflammation and neurodegeneration throughout lifetime. Population-based studies have previously shown an association between anemia and neurodegenerative disorders, such as Alzheimer's and Parkinson's disease $(71,72)$. This association is most evident when anemia onset occurs 20-29 years before Parkinson's disease (73). Irregular erythrocyte morphology and eryptosis has also been found to be enhanced in patients with Parkinson's disease (74).

The observations reported here might have a widespread clinical effect on future therapies targeting anemia. So far, EPO and its derivatives have been used to enhance erythropoiesis. EPO itself is controlled in an oxygen-dependent manner via the cellular oxygen-sensing axis HIF-2/PHD2 (75). At present, new inhibitors of PHD2 that in turn stabilize the $\alpha$-subunit of HIF-2 and subsequently increase transcription of the EPO gene (76) are in the process of being commercialized. As these small compounds will most probably cross the blood-brain barrier, it is conceivable that they will inhibit the PHD2 activity in neuronal cells too, ultimately increasing cerebral EPO production. If so, elevated EPO levels in the brain might have an effect on glucose metabolism and hypothalamic metabolism and inflammation, as shown here. A limitation of the current study is that the $T g 21$ mice, which have chronic overexpression of human EPO cDNA, can develop adaptive changes that could mask some potential neuroendocrine effects of EPO during DIO. These can be further investigated by studying a conditional overexpression system, which can eliminate the scope of any possible adaptive changes in $T g 21$ mice.

In summary, our studies demonstrate involvement of EPO/EpoR signaling in the brain and, particularly, in the hypothalamus in metabolic regulation during NCD and DIO. EPO overexpression in brain improves glucose utilization in male and female mice, possibly through Fgf2 1 and adiponectin regulation. On the other hand, EPO can exert an antiinflammatory response during DIO in male mice that effectively reduces microglial activation and recruitment of peripheral myeloid cells in the hypothalamus. In female mice, the antiobesity and hypothalamus antiinflammatory estrogen effects are predominant and the antiinflammatory effect of EPO is minimal, and the absence of estrogen's protective effect (via ovariectomy) allows for this EPO response. This also parallels EPO's regulation of weight and fat gain that we saw in male $T g 21$ mice but not in female $T g 21$ mice, which can only be seen in female mice after ovariectomy. These findings on activity associated with elevated EPO without increased EPO-stimulated erythropoiesis reveal a previously unrecognized EPO function in the brain that controls metabolism via central and peripheral action.

\section{Methods}

Mouse models. Tg21 mice, overexpressing PDGF $\beta$ promoter-driven human EPO (77), and WT mice (National Cancer Institute/Charles River Laboratories) were on a C57BL/6 background. The $T g 21$ mice were bred to homozygosity and verified by crossing with WT mice to check for all heterozygous pups. Deletion of the EpoR gene in neuronal cell precursors was achieved by nestin promoter-driven Cre recombinase expression in EpoR $R^{\text {loxP/loxP }}$ mice (22). The nestin-Cre mice, on a C57BL/6 background, were purchased from The Jackson Laboratory (stock no. 003771). EpoR ${ }^{\text {loxP/loxP }}$ mice were from a C57/129 hybrid mouse strain but were backcrossed onto a pure C57BL/6 background for at least 7 generations in our lab (78). Animals were housed in a specific pathogen-free and thermostable environment, with a 12-hour light/dark cycle and free access to food (NIH-31, 14\% kcal/fat, $3.0 \mathrm{kcal} / \mathrm{g}$, Teklad Diets) and water. For DIO, mice were fed HFD (60 kcal\% fat, 34.9\% crude fat, $5.24 \mathrm{kcal} / \mathrm{kg}$, Research Diets Inc.). Body composition, glucose tolerance, food intake, activity, hematocrit, and serum cytokine levels were assessed, and select tissues were harvested for gene and protein expression level. 
Immunofluorescence. Anesthetized mice were perfused with saline and 4\% paraformaldehyde in 100 $\mathrm{mM}$ phosphate buffer, and brains were dissected, postfixed in the same fixative overnight $\left(4^{\circ} \mathrm{C}\right)$, and immersed successively in $10 \%, 20 \%$, and $30 \%$ sucrose for 3 consecutive nights $\left(4^{\circ} \mathrm{C}\right)$. Hypothalami were separated from other regions, embedded in optimal cutting temperature compound, immediately frozen on dry ice, and stored at $-80^{\circ} \mathrm{C}$. Hypothalamic coronal sections $(10-\mu \mathrm{M}$ thick, 2 sections per slide) across the $\mathrm{MBH}$ were cut on a cryostat, blocked for 1 hour with $5 \%$ normal goat serum and $0.3 \%$ Triton X-100, and incubated with primary antibodies overnight at $4{ }^{\circ} \mathrm{C}$. Primary antibodies used were as follows: Iba1 (NCNP24, Wako Chemicals), TNF- $\alpha$ (ab1793, Abcam), CD169 and CD68 (MCA884 and MCA1957, respectively, Bio-Rad), and P2Y12 (AS-55043A, AnaSpec). Alexa Fluor-conjugated secondary antibodies were used for immunofluorescence microscopy. Sections were mounted with ProLong Gold antifade reagent with DAPI (Cell Signaling Technology) to identify cell nuclei. A Zeiss LSM 780 confocal scan head (Carl Zeiss Microscopy) mounted on a Zeiss AxioObserver.Z1 microscope, running ZEN 2.3 software, was used to acquire images at magnifications specified in the figure legends. The number of stain ${ }^{+}$ cells was counted in 4-6 microscopic fields of view of equal size in each sample. Cells were evaluated for the presence of a labeled nucleus, and immunofluorescently labeled cells were counted manually from 5-6 matched sections within prespecified regions of interest in the MBH using ImageJ software (NIH). For immunofluorescent intensity measurement using ImageJ, cells of interest in a field were selected using the drawing/selection tools. Area size, integrated density, and mean gray value were measured for immunofluorescent signal and background region. Corrected cell fluorescence intensity was measured by subtracting the product of area and mean fluorescence of background reading from integrated density. This was repeated for every cell in the field of view and repeated for 4-6 fields of view per slide and matched sections in the subsequent slide of same group. Data were then pooled for each group and graphically represented after statistical analysis.

Intracerebroventricular EPO infusion. Under isoflurane anesthesia, each mouse received a cannula (Brain Infusion Kit 3, Alzet) implanted over the appropriate stereotaxic coordinates: midline, $1.00 \mathrm{~mm}$; anteroposterior, $0.34 \mathrm{~mm}$; dorsoventral, $2.30 \mathrm{~mm}$. The cannula was fixed onto the skull with dental cement and connected via polyethylene tubing to a subcutaneously implanted 14-day osmotic minipump (Alzet, model 2006). Minipumps were filled with either recombinant human EPO (Epogen, Amgen) or saline.

Body composition and glucose tolerance test. Body weight was measured using the same balance by the same person. Body composition for fat mass and lean mass were measured using an EchoMRI 100H instrument (Echo Medical Systems) in conscious, immobilized mice. For glucose tolerance test, mice fasted overnight were injected with glucose $(2.0 \mathrm{~g} / \mathrm{kg})$. Blood glucose levels were measured before ( 0 minute) and up to 120 minutes after injection from tail vein bleeds (Alphatrak 2 glucometer, Zoetis). For determination of insulin secretion in response to glucose stimulation, $30 \mu \mathrm{l}$ blood was collected before $(0 \mathrm{~min})$ and after 120 minutes of glucose stimulation; then, serum was prepared and flash frozen in liquid nitrogen for later assessment.

Food intake, activity, and energy expenditure. Mice were allowed to adapt to individual housing for 2 weeks, and food and water were provided ad libitum. Food intake was then measured for 3 weeks. Energy expenditure was calculated using the energy balance method previously described (79). In brief, caloric energy intake was calculated from food intake, and change in body energy stores was calculated at $9.4 \mathrm{kcal} / \mathrm{g}$ of body fat and $1.0 \mathrm{kcal} / \mathrm{g}$ of fat-free mass. Energy expenditure was determined as the difference of caloric energy intake and sum of change in body energy store.

Real-time quantitative PCR. Total RNA was extracted using the RNeasy Lipid tissue mini kit (74804, Qiagen) according to manufacturer's instructions and reverse-transcribed with MuLVReverse Transcriptase (N8080018, Applied Biosystems). Transcript levels were measured using the semiquantitative realtime PCR on Quantstudio 7 Flex system (Applied Biosystems) using SYBR green detection of amplicons. Gene-specific primer sequences were as follows (forward and reverse, respectively): Ccl2 Fwd: CAGATGCAGTTAACGCCCCA, Rev: TGAGCTTGGTGACAAAAACTACAG; Il6 Fwd: AGCCAGAGTCCTTCAGAGAGAT, Rev: GAGAGCATTGGAAATTGGGGT; $I l 1 b$ Fwd: TGCCACCTTTTGACAGTGATG, Rev: TGATGTGCTGCTGCGAGATT; Illa Fwd: AGGGAGTCAACTCATTGGCG, Rev: TGGCAGAACTGTAGTCTTCGT; Ill0 Fwd: GCCAGAGCCACATGCTCCTA, Rev: TTTCCGATAAGGCTTGGCAAC; Socs3 Fwd: TAGACTTCACGGCTGCCAAC, Rev: CGgGGAGCTAGTCCCGAA; TNFa Fwd: TAGCCCACGTCGTAGCAAAC, Rev: ACAAGGTACAACCCATCGGC; $C R F$ Fwd: GGCATCCTGAGAGAAGTCCC, Rev: TGTTAGGGGCGCTCTCTTCT; Gapdh Fwd: CCCTTAAGAGGGATGCTGCC, Rev: TACGGCCAAATCCGTTCACA. 
Serum sample collection and cytokine measurements. Blood samples were collected by mandibular bleeding between $8 \mathrm{AM}$ and $9 \mathrm{AM}$. For serum preparation, blood was allowed to clot for 20-30 minutes at room temperature and then centrifuged at $2000 \mathrm{~g}$ for 20 minutes. Mice were fasted overnight before Fgf2 1 measurements. ELISA based assays were used to measure ACTH (RK-001-21, Phoenix Pharmaceuticals), corticosterone (ADI-900-097, Enzo Life Sciences), C-reactive protein (ab157712, Abcam), and Fgf21 and Adiponectin (MF2100 and MRP300, R\&D Systems). Insulin was measured in serum samples obtained during glucose tolerance test by ELISA (90080, Crystal Chem).

Ovariectomy. Bilateral ovaries were removed from female mice at 11 weeks of age as previously described (18). Sham surgery was also carried out in age-matched mice. Animals were allowed 1 week for recovery and depletion of endogenous estrogen in OVX mice and then put on HFD from 12 to 18 weeks of age.

Statistics. Data were analyzed using 2-way ANOVA for repeated measures between mouse groups. Post hoc comparison were made by Student's $t$ test (2-tailed) for continuous variables. A $\chi^{2}$ or Fisher's exact test was used for nominal variables, as appropriate followed by Bonferroni correction for multiple comparisons. $P$ values of less than 0.05 were considered significant.

Study approval. All animal protocols followed the NIH guidelines and had institution Animal Care and Use Committee approval (ACUC of the National Institute of Diabetes and Digestive and Kidney Diseases, $\mathrm{NIH}$, Bethesda, MD, USA).

\section{Author contributions}

SD designed the experiments, conducted the studies, analyzed the data, and wrote the manuscript. ZC, OG, $\mathrm{XZ}$, and MG contributed to data generation and analysis and reviewed and edited the manuscript. CTN contributed to the experimental design and discussion of data and reviewed and edited the manuscript. SD is the guarantor of this work and, as such, had full access to all data in the study and takes responsibility for the integrity of the data and accuracy of the data analysis.

\section{Acknowledgments}

The authors thank the National Institute of Diabetes and Digestive and Kidney Diseases Advanced Light Microscopy \& Image Analysis Core for the use of its resources. This work was supported by the Intramural Research Program of the National Institute of Diabetes and Digestive and Kidney Diseases.

Address correspondence to: Constance T. Noguchi, 10 Center Drive MSC-1822, Building 10, Room 9N319, Bethesda, Maryland 20892-1822, USA. Phone: 301.496.1163; Email: connien@niddk.nih.gov

1. Franke K, Gassmann M, Wielockx B. Erythrocytosis: the HIF pathway in control. Blood. 2013;122(7):1122-1128.

2. Wu H, Liu X, Jaenisch R, Lodish HF. Generation of committed erythroid BFU-E and CFU-E progenitors does not require erythropoietin or the erythropoietin receptor. Cell. 1995;83(1):59-67.

3. Lin CS, Lim SK, D'Agati V, Costantini F. Differential effects of an erythropoietin receptor gene disruption on primitive and definitive erythropoiesis. Genes Dev. 1996;10(2):154-164.

4. Gassmann M, Heinicke K, Soliz J, Ogunshola OO. Non-erythroid functions of erythropoietin. Adv Exp Med Biol. 2003;543:323-330.

5. Noguchi CT, Wang L, Rogers HM, Teng R, Jia Y. Survival and proliferative roles of erythropoietin beyond the erythroid lineage. Expert Rev Mol Med. 2008;10:e36.

6. Alnaeeli M, Raaka BM, Gavrilova O, Teng R, Chanturiya T, Noguchi CT. Erythropoietin signaling: a novel regulator of white adipose tissue inflammation during diet-induced obesity. Diabetes. 2014;63(7):2415-2431.

7. Nairz M, et al. Erythropoietin contrastingly affects bacterial infection and experimental colitis by inhibiting nuclear factor- $\kappa$ B-inducible immune pathways. Immunity. 2011;34(1):61-74

8. Hiram-Bab S, et al. Erythropoietin directly stimulates osteoclast precursors and induces bone loss. FASEB J. 2015;29(5):1890-1900.

9. Bond WS, Rex TS. Evidence that erythropoietin modulates neuroinflammation through differential action on neurons, astrocytes, and microglia. Front Immunol. 2014;5:523.

10. Yu X, et al. Erythropoietin receptor signalling is required for normal brain development. Development. 2002;129(2):505-516.

11. Beleslin-Cokic BB, Cokic VP, Yu X, Weksler BB, Schechter AN, Noguchi CT. Erythropoietin and hypoxia stimulate erythropoietin receptor and nitric oxide production by endothelial cells. Blood. 2004;104(7):2073-2080.

12. Teng R, et al. Disrupted erythropoietin signalling promotes obesity and alters hypothalamus proopiomelanocortin production. Nat Commun. 2011;2:520.

13. Dey S, et al. Erythropoietin regulates POMC expression via STAT3 and potentiates leptin response. J Mol Endocrinol. 2016;56(2):55-67

14. Dey S, Scullen T, Noguchi CT. Erythropoietin negatively regulates pituitary ACTH secretion. Brain Res. 2015;1608:14-20.

15. Foskett A, Alnaeeli M, Wang L, Teng R, Noguchi CT. The effects of erythropoietin dose titration during high-fat diet-induced obesity. J Biomed Biotechnol. 2011;2011:373781. 
16. Katz O, et al. Erythropoietin treatment leads to reduced blood glucose levels and body mass: insights from murine models. J Endocrinol. 2010;205(1):87-95.

17. Reinhardt M, Dey S, Tom Noguchi C, Zhang Y, Krakoff J, Thearle MS. Non-hematopoietic effects of endogenous erythropoietin on lean mass and body weight regulation. Obesity (Silver Spring). 2016;24(7):1530-1536.

18. Zhang Y, Rogers HM, Zhang X, Noguchi CT. Sex difference in mouse metabolic response to erythropoietin. FASEB J. 2017;31(6):2661-2673.

19. Marti HH, et al. Detection of erythropoietin in human liquor: intrinsic erythropoietin production in the brain. Kidney Int. 1997;51(2):416-418.

20. Bernaudin M, et al. Neurons and astrocytes express EPO mRNA: oxygen-sensing mechanisms that involve the redox-state of the brain. Glia. 2000;30(3):271-278.

21. Shingo T, Sorokan ST, Shimazaki T, Weiss S. Erythropoietin regulates the in vitro and in vivo production of neuronal progenitors by mammalian forebrain neural stem cells. J Neurosci. 2001;21(24):9733-9743.

22. Tsai PT, et al. A critical role of erythropoietin receptor in neurogenesis and post-stroke recovery. J Neurosci. 2006;26(4):1269-1274.

23. Wang L, et al. Matrix metalloproteinase 2 (MMP2) and MMP9 secreted by erythropoietin-activated endothelial cells promote neural progenitor cell migration. J Neurosci. 2006;26(22):5996-6003.

24. Chen ZY, Asavaritikrai P, Prchal JT, Noguchi CT. Endogenous erythropoietin signaling is required for normal neural progenitor cell proliferation. J Biol Chem. 2007;282(35):25875-25883.

25. Dey S, Noguchi CT. Erythropoietin and hypothalamic-pituitary axis. Vitam Horm. 2017;105:101-120.

26. Yang L, Hotamisligil GS. Stressing the brain, fattening the body. Cell. 2008;135(1):20-22.

27. Valdearcos M, Xu AW, Koliwad SK. Hypothalamic inflammation in the control of metabolic function. Annu Rev Physiol. 2015;77:131-160.

28. Wiessner C, Allegrini PR, Ekatodramis D, Jewell UR, Stallmach T, Gassmann M. Increased cerebral infarct volumes in polyglobulic mice overexpressing erythropoietin. J Cereb Blood Flow Metab. 2001;21(7):857-864.

29. Valdearcos M, et al. Microglial inflammatory signaling orchestrates the hypothalamic immune response to dietary excess and mediates obesity susceptibility. Cell Metab. 2018;27(6):1356.

30. Bennett ML, et al. New tools for studying microglia in the mouse and human CNS. Proc Natl Acad Sci USA. 2016;113(12):E1738-E1746.

31. Butovsky O, et al. Modulating inflammatory monocytes with a unique microRNA gene signature ameliorates murine ALS J Clin Invest. 2012;122(9):3063-3087.

32. Tronche F, et al. Disruption of the glucocorticoid receptor gene in the nervous system results in reduced anxiety. Nat Genet. 1999;23(1):99-103.

33. Galichet C, Lovell-Badge R, Rizzoti K. Nestin-Cre mice are affected by hypopituitarism, which is not due to significant activity of the transgene in the pituitary gland. PLOS ONE. 2010;5(7):e11443.

34. Giusti SA, et al. Behavioral phenotyping of Nestin-Cre mice: implications for genetic mouse models of psychiatric disorders. J Psychiatr Res. 2014;55:87-95.

35. Harno E, Cottrell EC, White A. Metabolic pitfalls of CNS Cre-based technology. Cell Metab. 2013;18(1):21-28.

36. Lin Z, et al. Adiponectin mediates the metabolic effects of FGF21 on glucose homeostasis and insulin sensitivity in mice. Cell Metab. 2013;17(5):779-789.

37. Badman MK, Pissios P, Kennedy AR, Koukos G, Flier JS, Maratos-Flier E. Hepatic fibroblast growth factor 21 is regulated by PPARalpha and is a key mediator of hepatic lipid metabolism in ketotic states. Cell Metab. 2007;5(6):426-437.

38. Potthoff MJ, et al. FGF21 induces PGC-1alpha and regulates carbohydrate and fatty acid metabolism during the adaptive starvation response. Proc Natl Acad Sci USA. 2009;106(26):10853-10858.

39. Liang Q, et al. FGF21 maintains glucose homeostasis by mediating the cross talk between liver and brain during prolonged fasting. Diabetes. 2014;63(12):4064-4075.

40. Hanoun M, Maryanovich M, Arnal-Estapé A, Frenette PS. Neural regulation of hematopoiesis, inflammation, and cancer. Neuron. 2015;86(2):360-373.

41. Hong J, Stubbins RE, Smith RR, Harvey AE, Núñez NP. Differential susceptibility to obesity between male, female and ovariectomized female mice. Nutr J. 2009;8:11.

42. Dorfman MD, et al. Sex differences in microglial CX3CR1 signalling determine obesity susceptibility in mice. Nat Commun. 2017;8:14556

43. Morselli E, et al. Hypothalamic PGC-1 $\alpha$ protects against high-fat diet exposure by regulating ER $\alpha$. Cell Rep. 2014;9(2):633-645.

44. Argente-Arizón P, et al. The hypothalamic inflammatory/gliosis response to neonatal overnutrition is sex and age dependent. Endocrinology. 2018;159(1):368-387.

45. Brines ML, et al. Erythropoietin crosses the blood-brain barrier to protect against experimental brain injury. Proc Natl Acad Sci USA. 2000;97(19):10526-10531.

46. Ehrenreich H, et al. Erythropoietin: a candidate compound for neuroprotection in schizophrenia. Mol Psychiatry. 2004;9(1):42-54

47. Schuler B, Vogel J, Grenacher B, Jacobs RA, Arras M, Gassmann M. Acute and chronic elevation of erythropoietin in the brain improves exercise performance in mice without inducing erythropoiesis. FASEB J. 2012;26(9):3884-3890.

48. Sadamoto Y, et al. Erythropoietin prevents place navigation disability and cortical infarction in rats with permanent occlusion of the middle cerebral artery. Biochem Biophys Res Commun. 1998;253(1):26-32.

49. Sakanaka M, et al. In vivo evidence that erythropoietin protects neurons from ischemic damage. Proc Natl Acad Sci USA. 1998;95(8):4635-4640

50. Bernaudin M, et al. A potential role for erythropoietin in focal permanent cerebral ischemia in mice. J Cereb Blood Flow Metab. 1999;19(6):643-651.

51. Katayama Y, et al. Signals from the sympathetic nervous system regulate hematopoietic stem cell egress from bone marrow. Cell. 2006;124(2):407-421.

52. Méndez-Ferrer S, Lucas D, Battista M, Frenette PS. Haematopoietic stem cell release is regulated by circadian oscillations Nature. 2008;452(7186):442-447. 
53. Yamazaki S, et al. Nonmyelinating Schwann cells maintain hematopoietic stem cell hibernation in the bone marrow niche. Cell. 2011;147(5):1146-1158.

54. Markan KR, et al. Circulating FGF21 is liver derived and enhances glucose uptake during refeeding and overfeeding. Diabetes. 2014;63(12):4057-4063.

55. BonDurant LD, Potthoff MJ. Fibroblast growth factor 21: a versatile regulator of metabolic homeostasis. Annu Rev Nutr. 2018;38:173-196.

56. Zhang X, et al. Serum FGF21 levels are increased in obesity and are independently associated with the metabolic syndrome in humans. Diabetes. 2008;57(5):1246-1253.

57. Markan KR, Potthoff MJ. Metabolic fibroblast growth factors (FGFs): Mediators of energy homeostasis. Semin Cell Dev Biol. 2016;53:85-93.

58. Markan KR. Defining "FGF21 Resistance" during obesity: Controversy, criteria and unresolved questions. F1000Res. 2018;7:289.

59. Bookout AL, et al. FGF21 regulates metabolism and circadian behavior by acting on the nervous system. Nat Med. 2013;19(9):1147-1152.

60. Zhang X, Zhang G, Zhang H, Karin M, Bai H, Cai D. Hypothalamic IKKbeta/NF-kappaB and ER stress link overnutrition to energy imbalance and obesity. Cell. 2008;135(1):61-73.

61. Kleinridders A, et al. MyD88 signaling in the CNS is required for development of fatty acid-induced leptin resistance and diet-induced obesity. Cell Metab. 2009;10(4):249-259.

62. Milanski M, et al. Inhibition of hypothalamic inflammation reverses diet-induced insulin resistance in the liver. Diabetes. 2012;61(6):1455-1462.

63. Valdearcos M, et al. Microglial inflammatory signaling orchestrates the hypothalamic immune response to dietary excess and mediates obesity susceptibility. Cell Metab. 2017;26(1):185-197.e3.

64. Kulkarni S, et al. Adult enteric nervous system in health is maintained by a dynamic balance between neuronal apoptosis and neurogenesis. Proc Natl Acad Sci USA. 2017;114(18):E3709-E3718.

65. Bernal A, Arranz L. Nestin-expressing progenitor cells: function, identity and therapeutic implications. Cell Mol Life Sci. 2018;75(12):2177-2195

66. Orsi NM, Gopichandran N, Ekbote UV, Walker JJ. Murine serum cytokines throughout the estrous cycle, pregnancy and post partum period. Anim Reprod Sci. 2006;96(1-2):54-65.

67. Spinedi E, Suescun MO, Hadid R, Daneva T, Gaillard RC. Effects of gonadectomy and sex hormone therapy on the endotoxin-stimulated hypothalamo-pituitary-adrenal axis: evidence for a neuroendocrine-immunological sexual dimorphism. Endocrinology. 1992;131(5):2430-2436.

68. Soliz J, Thomsen JJ, Soulage C, Lundby C, Gassmann M. Sex-dependent regulation of hypoxic ventilation in mice and humans is mediated by erythropoietin. Am J Physiol Regul Integr Comp Physiol. 2009;296(6):R1837-R1846.

69. Gassmann M, Tissot van Patot M, Soliz J. The neuronal control of hypoxic ventilation: erythropoietin and sexual dimorphism. Ann N Y Acad Sci. 2009;1177:151-161.

70. Gassmann M, Pfistner C, Doan VD, Vogel J, Soliz J. Impaired ventilatory acclimatization to hypoxia in female mice overexpressing erythropoietin: unexpected deleterious effect of estradiol in carotid bodies. Am J Physiol Regul Integr Comp Physiol. 2010;299(6):R1511-R1520.

71. Hong CT, Huang YH, Liu HY, Chiou HY, Chan L, Chien LN. Newly diagnosed anemia increases risk of Parkinson's disease: a population-based cohort study. Sci Rep. 2016;6:29651.

72. Hong CH, et al. Anemia and risk of dementia in older adults: findings from the Health ABC study. Neurology. 2013;81(6):528-533.

73. Savica R, et al. Anemia or low hemoglobin levels preceding Parkinson disease: a case-control study. Neurology. 2009;73(17):1381-1387.

74. Pretorius E, Swanepoel AC, Buys AV, Vermeulen N, Duim W, Kell DB. Eryptosis as a marker of Parkinson's disease. Aging (Albany NY). 2014;6(10):788-819.

75. Gassmann M, Muckenthaler MU. Adaptation of iron requirement to hypoxic conditions at high altitude. J Appl Physiol. 2015;119(12):1432-1440.

76. Chen N, et al. Roxadustat treatment for anemia in patients undergoing long-term dialysis. N Engl J Med. 2019;381(11):1011-1022.

77. Ruschitzka FT, et al. Nitric oxide prevents cardiovascular disease and determines survival in polyglobulic mice overexpressing erythropoietin. Proc Natl Acad Sci USA. 2000;97(21):11609-11613.

78. Wang L, et al. PPAR $\alpha$ and Sirt1 mediate erythropoietin action in increasing metabolic activity and browning of white adipocytes to protect against obesity and metabolic disorders. Diabetes. 2013;62(12):4122-4131.

79. Ravussin Y, Gutman R, LeDuc CA, Leibel RL. Estimating energy expenditure in mice using an energy balance technique. Int $J$ Obes (Lond). 2013;37(3):399-403 